\title{
Longwave band-by-band cloud radiative effect and its application in GCM evaluation
}

\author{
Xianglei Huang ${ }^{1 *}$, Jason N.S. Cole ${ }^{2}$, Fei He ${ }^{1}$, Gerald L. Potter ${ }^{1}$, Lazaros Oreopoulos ${ }^{3}$, Dongmin
} Lee $^{3,4}$, Max Suarez ${ }^{3}$, Norman G. Loeb ${ }^{5}$

1. Dept. of Atmos., Oceanic, and Space Sciences, University of Michigan, Ann Arbor, Michigan

2. Canadian Centre for Climate Modeling and Analysis, Environment Canada, Toronto, Ontario, Canada

3. NASA Goddard Space Flight Center, Greenbelt, Maryland

4. Climate Dynamics Laboratory, Seoul National University, Seoul, Korea

5. Radiation and Climate Branch, NASA Langley Research Center, Hampton, Virginia

* Corresponding author: Prof. Xianglei Huang, Dept. of Atmos., Oceanic, and Space Sciences, University of Michigan, Ann Arbor, MI 48109-2143, xianglei@umich.edu, (734)936-0491.

Submitted to Journal of Climate

February 7, 2012 


\section{Abstract}

The cloud radiative effect (CRE) of each longwave (LW) absorption band of a GCM's radiation code is uniquely valuable for GCM evaluation because (1) comparing band-by-band CRE avoids the compensating biases in the broadband CRE comparison and (2) the fractional contribution of each band to the LW broadband CRE ( $\left.f_{\text {CRE }}\right)$ is sensitive to cloud top height but largely insensitive to cloud fraction, presenting thus a diagnostic metric to separate the two macroscopic properties of clouds. Recent studies led by the first author have established methods to derive such band-by-band quantities from collocated AIRS and CERES observations. We present here a study that compares the observed band-by-band CRE over the tropical oceans with those simulated by three different atmospheric GCMs (GFDL AM2, NASA GEOS-5, and CCCma CanAM4) forced by observed SST. The models agree with observation on the annual-mean LW broadband CRE over the tropical oceans within $\pm 1 \mathrm{Wm}^{-2}$. However, the differences among these three GCMs in some bands can be as large as or even larger than $\pm 1 \mathrm{Wm}^{-2}$. Observed seasonal cycles of $\mathrm{f}_{\mathrm{CRE}}$ in major bands are shown to be consistent with the seasonal cycle of cloud top pressure for both the amplitude and the phase. However, while the three simulated seasonal cycles of $f_{\text {CRE }}$ agree with observations on the phase, the amplitudes are underestimated. Simulated interannual anomalies from GFDL AM2 and CCCma CanAM4 are in phase with observed anomalies. The spatial distribution of $f_{C R E}$ highlights the discrepancies between models and observation over the low-cloud regions and the compensating biases from different bands. 


\section{Introduction}

Since the 1980s, broadband radiative flux and cloud radiative effect (CRE, the difference between all-sky and clear-sky fluxes), have been extensively used in climate studies (e.g. Ramanathan et al. 1989; Wielicki et al. 2002; Wong et al. 2006), especially in the evaluation of climate models and cloud feedback studies (e.g. Allan et al. 2004; Allan \& Ringer 2003; Raval et al. 1994; Slingo et al. 1998; Wielicki et al. 2002; Yang et al. 1999). In the development of a GCM for climate studies, one inevitable and important step is "tuning" in which poorly constrained parameters are adjusted using observations and physical principles to ensure energy balance at the top of atmosphere (TOA). This ensures that simulated broadband quantities are generally consistent with the observed counterparts at the TOA. However, it cannot guarantee the consistency between band-by-band decompositions of observed and simulated radiation fluxes. In fact quite often the compensating biases from different absorption bands offset each other and lead to a seemingly good agreement between modeled and observed broadband fluxes (e.g., see the work in the thermal infrared by Huang et al. 2006; Huang et al. 2007; Huang et al. 2008; and Huang et al. 2010). Similar compensating biases can be expected in the simulated broadband CRE as well. Therefore, directly using of band-by-band flux and CRE in model evaluation can avoid the compensating errors and highlight the biases in different bands since flux and CRE of each individual molecular absorption band are calculated directly in the GCM radiation scheme. Moreover, as illustrated in Huang et al. (2010) with both conceptual model and numerical simulation, the fractional contribution of each band to the broadband longwave CRE (for brevity, hereafter denoted as $f_{C R E}$ ) is sensitive to cloud top height but largely insensitive to cloud fraction. The LW broadband CRE can be written as $C R E_{L W}=f c\left(F_{c l r}-F_{c l d}\right)$ where $F$ is the 
outgoing longwave flux at top of atmosphere, $\mathrm{fc}$ is the cloud fraction, and subscripts clr and cld denote clear-sky and overcast cloudy sky, respecitvely. Correspondingly, the i-th band CRE can be written as $C R E^{i}=f c\left(F_{c l r}^{i}-F_{c l d}^{i}\right)$, and the superscript ${ }^{i}$ denotes the i-th band. The resulting expression for $f_{C R E}$ is,

$$
f_{C R E}=\frac{F^{i}{ }_{c l r}-F^{i}{ }_{c l d}}{F_{c l r}-F_{c l d}}
$$

Therefore, the common factor of cloud fraction cancels and $f_{C R E}$ is only sensitive to cloud top temperature, making $\mathrm{f}_{\mathrm{CRE}}$ a useful quantity in diagnosing and evaluating modeled CRE. The LW broadband CRE is sensitive to both the cloud fraction and (mostly) cloud top height while the SW broadband CRE is sensitive to both the cloud fraction and (mostly) the cloud water path (i.e. cloud reflectance). Since the $L W f_{C R E}$ is sensitive to cloud top height but not to cloud fraction, it provides a dimension to sort out the contributions of cloud faction and cloud top height to the broadband CRE.

Huang et al. (2008) and Huang et al. (2010) mainly focused on the algorithm for deriving such band-by-band LW flux and CRE from the AIRS spectral radiances collocated with CERES ${ }^{1}$ observations from the same Aqua spacecraft. Using one year of data and corresponding simulations from a GFDL AGCM (AM2), both studies also showed preliminary usage of such data in GCM evaluation. To further explore and demonstrate the potential of such band-by-band CRE in model evaluations, this study performs the first ever comprehensive evaluations of GCMsimulated band-by-band CRE from three different GCMs. Multiple years of data are used to

\footnotetext{
${ }^{1}$ AIRS stands for Atmospheric Infrared Sounder, which is a grating spectrometer. CERES stands for Clouds and Earth's Radiant Energy System, which consists of two broadband radiometers and one narrow-band radiometer.
} 
explore the long-term mean, seasonal cycles, as well as interannual variations of CRE and $f_{C R E}$ of each LW band.

The data and GCMs used in this study are described in Section 2. Sections 3 and 4 present the comparison results. Conclusion and further discussion are given in Section 5.

\section{Observations and GCM simulations}

\subsection{Observations}

The algorithms in Huang et al. (2008) and Huang et al. (2010) were developed for and validated against observations over the tropical open oceans $\left(30^{\circ} \mathrm{S}\right.$ to $\left.30^{\circ} \mathrm{N}\right)$. Hence here we employ the algorithms to derive clear-sky and all-sky spectral fluxes at a $10 \mathrm{~cm}^{-1}$ interval for the longwave spectrum $\left(0-2000 \mathrm{~cm}^{-1}\right)$ using the collocated AIRS and Aqua-CERES observations over the tropical open oceans from 2003 to 2007 . The monthly mean spectral CRE is then calculated in a similar way as the broadband CRE derived from ERBE or CERES observations. The scene type information from CERES SSF (Single Satellite Footprint) is used with pre-developed spectral anisotropic distribution model (ADM) to invert spectral flux for each AIRS channel, and a multilinear regression scheme is then used to estimate the spectral flux over spectral region not covered by the AIRS channels. As shown in Huang et al. (2008) and Huang et al. (2010), the OLR (outgoing longwave radiation) derived by this approach agrees well with the collocated CERES OLR and this good agreement is found over different cloud scene types (as distinguished by distinct cloud fractions and cloud-surface temperature contrasts). Comparisons with synthetic data showed that the algorithm can reliably estimate spectral flux at $10 \mathrm{~cm}^{-1}$ resolution with maximum fractional difference less than $\pm 5 \%$ for clear-sky scenes and $\sim \pm 3.6 \%$ for cloudy-sky 
scenes. Details of the algorithm and validation can be found in Huang et al. (2008) and Huang et al. (2010).

Table 1 summarizes the comparison between OLR derived by this spectral ADM approach with the collocated CERES OLR for 2003 to 2007 . AIRS version5 calibrated radiances and CERES SSF (single satellite footprint) Edition 2 data are used. The $2 \sigma$ radiometric calibration uncertainty of CERES OLR is $\sim 1 \%$, which translates to $\sim 2.5 \mathrm{Wm}^{-2}$ for a typical OLR value in the tropics. Table1 shows that, for all the years, the mean difference between CERES clear-sky OLR and our derived clear-sky OLR is always below the $2 \sigma$ uncertainty. The cloudy-sky OLR mean difference is about the same as the $2 \sigma$ uncertainty. The standard deviations of these differences change little from year to year.

\subsection{GCMs}

We used three atmospheric general circulation models (hereafter, GCM) in this study, the GFDL AM2 model (GFDL GAMDT 2004), the Fortuna 2.2 version of the NASA GEOS-5 model (Molod et al. 2012), and the fourth generation AGCM at CCCma, Environment Canada (CanAM4) (vonSalzen et. al. 2012, manuscript submitted to Atmosphere-Ocean). Each GCM is forced with observed SSTs over multiple years. For each model, clear-sky flux and all-sky fluxes of the individual bands of the LW radiative transfer code are directly saved in the output of simulations. For GEOS-5 and CanAM4, the simulations were carried out for 2000-2009 while only the 2003-2007 period is analyzed and compared with observations here. For AM2, due to the limited resources, the simulation was carried out only up to 2005 so only the simulations from 2003 to 2005 are analyzed here. As will be shown in the next section, the year-to-year 
variations of band-by-band $C R E$ and $f_{C R E}$ are small so the different period in the $A M 2$ simulations has little effect on the comparisons of long-term mean and mean seasonal cycle.

The longwave radiation scheme in the GFDL AM2 model follows Schwarzkopf \& Ramaswamy (1999). The LW spectrum is divided into eight bands, with two water bands (far-IR and $>1400 \mathrm{~cm}^{-1}$ ) treated together, in practice. Clouds are assumed to be non-scattering in the LW. The LW radiation scheme in the NASA GEOS-5 is based on Chou et al. (2003). It divides the LW spectrum into 9 bands and can be run at two accuracy modes (high accuracy mode is used here) using either a k-distribution or look-up table approach to calculate gaseous transmission functions. Cloud scattering in the LW is handled empirically using a rescaling approach. In the CanAM4 model, the optical properties of gases is modeled using a correlated- $k$ distribution (Li and Barker 2005) while the radiative transfer is simulated using the Monte Carlo Independent Column Approximation and two-stream radiative transfer solutions (von Salzen et al. 2012, manuscript submitted to Atmosphere-Ocean; Pincus et. al. 2003; Li 2002). Scattering by clouds droplets is included based on the absorption approximation (Li and Fu, 2000). Note both GEOS5 and CanAM4 models take into account the scattering of cloud in the longwave.

\subsection{Redefining the bands for comparison}

Because of the different radiation schemes used in the three GCMs, the number of bands and the bandwidth for each band are not necessarily the same. In fact, GFDL AM2 uses 8 bands in the LW while NASA GEOS-5 used 10 bands and CanAM4 uses 9 (Table 2). To facilitate the comparison, we define five new bands (Table 2) to be used for comparisons between GCMs and between GCMs and observations, each of which is either a band common to the GCM radiative transfer schemes or some combination of bands used in the GCMS. This ensures the 
maximum compatibility among GCMs for the band-by-band comparisons. As shown in Table 2, after merging into five bands, the bandwidth structures of GEOS-5 and CanAM4 models are for the most part consistent with each other and consistent with the five bands defined for the observational analysis. AM2 bandwidths are slightly different. To understand the effect of such intrinsic differences in the bandwidths in the GCMs on the band-by-band fluxes and CREs, we compute the flux of each band $\left(F_{\text {model_bnd }}\right)$ for a blackbody at a temperature $T$ using the bandwidth from each GCM. This flux is then compared to the flux of the band defined for this study $\left(F_{b n d}\right)$. The relative difference, $\left(F_{\text {model_bnd }}-F_{b n d}\right) / F_{b n d}$, is shown in Fig. 1 for $T=220 \mathrm{~K}$ and $T=$ 298K, respectively. Except for Band4 (ozone band, $980-1100 \mathrm{~cm}^{-1}$ ) and Band5 $\left(1100-1400 \mathrm{~cm}^{-1}\right.$ ), the relative difference due to the varying bandwidths is within $10 \%$ (Figure1a and $1 \mathrm{~b}$ ). By subtracting the flux for $T=298 \mathrm{~K}$ from the flux for $\mathrm{T}=220 \mathrm{~K}$, which mimics the magnitude of LW CRE (Fig. 1c), we find that the relative difference for each band in each GCM's radiation scheme is about the same. The largest relative difference is $\sim 33 \%$ and always occurs in the ozone band. This larger error occurs because of the rapid decrease of the blackbody curve between 220 and 298K over this band, which causes the small bandwidth differences between AM2 and GEOS5/CanAM4 to lead to large fractional difference in flux. For this reason, we omit here the ozone band (band4) in some analyses presented in Sections 3 and 4. If an ozone-band result is discussed, it must be interpreted with caution for the GFDL AM2 which has a different bandwidth for the band from the other two GCMs and the observations. For most of the analysis, our focus is primarily on band1, band2, and band3, for which the intrinsic bandwidth differences in the GCMs yield no more than $10 \%$ relative difference in the flux, CRE, and $\mathrm{f}_{\mathrm{CRE}}$. 


\section{Model intercomparisons and comparisons with observations: averages over the tropical}

ocean

Figure 2 shows the five-year averages of all-sky OLR and LW CRE from the NASA GEOS-5 and CCCma CanAM4 model simulations, three-year averages from the GFDL AM2 simulation, and the counterpart 5-year averages from the collocated AIRS and CERES observations (hereafter, referred to as observations for the brevity). Generally the models and the observations are consistent with each other on the broad spatial features of both all-sky OLR and longwave CRE. In the tropical Pacific warm pool east of the Maritime Continent, the AM2 and CanAM4 models have lower all-sky OLR than the observation and the GEOS-5 model. Accordingly, their LW CREs are higher than those in the observations and the GEOS-5 model. The LW CRE is only $\sim 10 \mathrm{Wm}^{-2}$ (or even smaller) over the oceans west of major continents because these regions are frequently covered by marine stratus with cloud top $\sim 1 \mathrm{~km}$ and thus has little thermal contrast with surface. Judging from the LW broadband CRE here alone, observations and all GCMs seemingly agrees better over the low-cloud regions than over the high-cloud regions such as the ITCZ and SPCZ (Southern Pacific Convergence Zone). However, as we shall show in the following subsections, the $f_{C R E}$ over the low-cloud regions is indeed quite different among the GCMs and between the models and observation.

\subsection{Long-term means and seasonal cycles}

Table 3 summarizes the multi-year means of the LW broadband and band-by-band CREs averaged over the entire tropical oceans. Observed long-term mean LW broadband CRE is 28.5 $\mathrm{Wm}^{-2}$ while the model results are slightly lower by $0.2-1.2 \mathrm{Wm}^{-2}$. However, the difference in the CRE of a particular band could be easily as large as, or even larger than, the broadband 
difference. For example, the differences between observed and simulated CREs in Band2 (the $\mathrm{CO}_{2}$ band) are $\sim-0.6$ to $0.7 \mathrm{Wm}^{-2}$. The difference between observed and CanAM4-simulated CRE in Band1 alone is $\sim 1.12 \mathrm{Wm}^{-2}$. Modeled broadband CREs are always smaller than the observed CRE, but modeled CREs in a given band could be either larger or smaller than its observed counterpart. Such compensation among bands leads to an apparent good agreement of the broadband LW CRE. Band-by-band CREs exposes such compensation in a quantitative way and makes it possible to further examine the sources of the compensating biases.

Among the five bands examined here, the largest contributor to LW CRE is Band3, the first window region from 800 to $980 \mathrm{~cm}^{-1}$, and is responsible for $\sim 32-36 \%$ of LW CRE. There is a large discrepancy in the CRE of Band4 (ozone band) between the AM2 model and the other two models and the observations. As mentioned in Section 2, the AM2 model has a different bandwidth for Band4 while others have identical bandwidth. Second, the AM2 simulation here is done with prescribed climatological ozone profiles at the 1990 s level obtained from a combination of ozonesonde and satellite measurements (Paul et al. 1998; GFDL GAMDT 2004). In contrast, the GEOS-5 model ozone fields are calculated on-line inside the GCM using a parameterization described in Rienecker et al. (2008). The CanAM4 simulation used a zonally averaged version of AC\&C/SPARC ozone database that was prepared for the CMIP5 (Coupledmodel inter-comparison project, phase 5) model simulations (Cionni et al. 2011). Over the historical period, the database consists of time-varying ozone fields based on observations for the stratosphere and chemistry-climate-model simulations for the troposphere. Band 4, the ozone band, never saturates, which makes it sensitive to stratospheric and tropospheric ozone as well as to surface and cloud temperatures. Moreover, ozone spatial distribution is indeed 
affected by the large-scale circulation and transport. Therefore, besides the band-width discrepancies, whether realistic on-line ozone fields are available or not could potentially affect the simulated CRE results as well.

Seasonal cycles of CREs of Bands 1-3 are shown in Fig. 3. Compared to the mean values shown in Table 3, the seasonal fluctuation is very small ( $3.5 \%$ or even smaller). For both models and observations, each band CRE seasonal cycle closely tracks one another. The observations show two peaks with one in April-May and the other in November-December, which is related to the movement of the Sun as well as the north-south seasonal movement of ITCZ. The models also show such semi-seasonal cycles with similar phase except that the first peak in the GEOS-5 is one month ahead of the observations. Although the phases are generally consistent to each other, the magnitudes of the seasonal cycles are noticeably different by about a factor of two, with the observations and GEOS- 5 having similar amplitudes, while those of AM2 and CanAM4 being much smaller.

The good phase syncing between CREs of different bands is largely due to the fact that for all the LW bands the surface and cloud top temperature contrast is the largest driver of CRE. Therefore, they all vary in phase with the LW broadband CRE in terms of the absolute magnitude. Seasonal cycle of the $\mathrm{f}_{\mathrm{CRE}}$ (Fig. 4) is different because, in this way, the LW broadband CRE is always normalized to $100 \%$ for each month. In contrast to the seasonal cycle of absolute band CRE (Fig. 3), for both models and observations a single annual cycle is dominant instead of a semi-annual cycle. The seasonal cycle of Band1 (water-vapor band) $f_{C R E}$ is strongly anticorrelated with that of Band3 (the window region) $f_{\text {CRE. }}$. For both models and the observation, Band1 peaks in March-April while Band3 peaks in July-August. This suggests that any phase 
discrepancies shown in Fig. 3 are likely dominated by differences in cloud fraction rather than differences in cloud top height (otherwise, the same discrepancies would have appeared in such $f_{C R E}$ seasonal cycles as well). For $f_{C R E}$ seasonal cycles, the $G C M$ s have similar amplitudes but are 2-3 times smaller than the observed amplitude (Fig. 4).

Figure 4 also shows that the seasonal cycle of Band $2 f_{\text {CRE }}$ closely tracks that of Band 1 but anti-correlated with that of Band3. Figure 5 plots the correlation coefficients between the seasonal cycle of Band $1 f_{C R E}$ and the seasonal cycle of $f_{C R E}$ of the remaining bands. Models and observations behave similarly in terms of such inter-band correlations: window bands (Band3 and Band5) are strongly anti-correlated with Band1. The observations exhibit a moderate positive correlation between Band2 and Band1 while much stronger positive correlations are seen for the GCMs. The largest discrepancies exist in Band4 (the ozone band). As expected, Band4 $f_{C R E}$ in observation, GEOS-5, and CanAM4 shows strong in-phase relation with Band3 $f_{C R E \text {, }}$ since cloud and surface thermal contrast significantly affects both bands. For the AM2 model, the correlation between Band4 and Band3 $f_{C R E}$ is weak, partly due to the prescribed climatological ozone profiles. Such differences indicate the benefit of having realistic or selfconsistent ozone profiles in the transient simulations.

\subsection{Explanations of the seasonal cycles}

To understand the seasonal cycles shown in Figs. 3-4, it is instructive to look the seasonality of cloud macroscopic properties averaged over the tropical oceans. Fig. 6(a) shows the ISCCP (International Satellite Cloud Climatology Project, Rossow \& Schiffer 1991; Rossow \& Schiffer 1999) long-term mean seasonal cycles of cloud fractions for high, middle, and low cloud, respectively. The peaks of high-cloud fraction can be found during two periods: April-May and 
November-January, the latter being also the peak for middle-cloud fraction. The seasonality of high-cloud and middle-cloud fraction is consistent with the observed seasonal cycles of bandby-band CRE in Fig. 3. Meanwhile, even though the low-cloud fraction peaks in July-August, it contributes little to the observed seasonal cycle of CRE because low clouds contribute little to the absolute LW CRE or the absolute band CRE.

Fig. $6 \mathrm{~b}$ shows the ISCCP mean seasonal cycle of cloud top pressure (CTP) averaged over the tropical oceans. In contrast to the seasonality of cloud fractions, it shows that mean cloud top is highest in April-May and lowest in August-September. As shown in Fig. 7b in Huang et al. (2010), the higher the cloud top, the larger the Band1 contribution to the total LW CRE and the smaller the Band3 contribution. This behavior is mainly due to the shift of blackbody peak emission towards lower frequency as the cloud top temperature becomes colder (i.e. Wien's displacement law). Therefore, the seasonality of CTP is consistent with the observed seasonality of $f_{C R E}$ in Fig. 4.

Given the strong anti-correlation between Band1 $\mathrm{f}_{\mathrm{CRE}}$ and Band $3 \mathrm{f}_{\mathrm{CRE}}$, it is worthwhile to explore to what extent we can use the ISCCP mean seasonal cycle of CTP to estimate the amplitudes of the seasonal cycles of Band1 and Band3. For both seasonal cycles, we use the standard deviation as a measure of the amplitude. Similar to Huang et al. (2010), we assume a layer of optically thick cloud ( $\tau>>1)$ and typical tropical sounding profiles of temperature, humidity, and ozone (McClatchey 1972). Then we vary the cloud top from the lower troposphere to the upper troposphere and use MODTRAN5 (Berk et al., 2005) to calculate $f_{\mathrm{CRE}}$ of Band1 and Band3 accordingly (Fig. 7a). When the cloud top is lower than $600 \mathrm{hPa}$, Band1 $\mathrm{f}_{\mathrm{CRE}}$ is less than $\sim 6 \%$ while Band3 $\mathrm{f}_{\mathrm{CRE}}$ is more than $40 \%$. As the cloud top moves upward, the 
contribution from Band1 gradually increases while that from Band3 decreases. When the cloud top reaches $150 \mathrm{hPa}$, the contributions from both bands are essentially equal ( $\left.f_{\mathrm{CRE}} \sim 28 \%\right)$. Fig. $7 \mathrm{a}$ also shows that, above $600 \mathrm{hPa}$, the change of the fractional contribution with respect to cloud top pressure is nearly linear. Therefore, we define the linear regression slope for the $i$-th band as

$$
r_{i}=\frac{d f_{C R E}^{i}}{d C T P}
$$

where superscript $i$ denotes the $i$-th band. $r_{1}$ and $r_{3}$ are then derived by linear regression of the curve shown in Fig. 7a. Then the amplitude of seasonal cycle of Band1 (Band3) can then be estimated by multiplying $r 1(r 3)$ with the standard deviation of ISCCP CTP seasonal cycle in Fig. $6 \mathrm{~b}$, which is $12.2 \mathrm{hPa}$. Fig. $7 \mathrm{~b}$ shows the amplitudes estimated in this manner vs. the real amplitudes derived from Fig. 4 top panel. The simple estimations are $82 \%$ and $85 \%$ of the observed values for Band1 and Band3, respectively. No cloud fraction values are involved in this estimation, which corroborates the fact that the fractional contribution of each band is largely insensitive to cloud fraction but is mainly affected by changes in cloud top pressure. This behavior is very different from that of the absolute amount of band-by-band CRE, which is largely influenced by the fractions of high cloud and middle clouds.

Since the ISCCP CTP seasonality can largely explain the observed seasonality of $f_{\mathrm{CRE}}$, the model-observation discrepancies shown in Fig.4 should be largely due to the difference in observed and simulated seasonal cycles of CTP, especially the amplitudes. Using the same slopes of $r_{1}$ and $r_{3}$ derived above, we estimated the amplitudes (the standard deviations) of CTP 
seasonal cycles for AM2, GEOS-5, and CanAM4 models are $2.9 \mathrm{hPa}, 3.7 \mathrm{hPa}, 4.7 \mathrm{hPa}$, respectively (in comparison to the $12.2 \mathrm{hPa}$ derived from ISCCP's mean CTP seasonal cycle).

\subsection{Interannual variations}

Figure 8 shows the 5 -month running means of deseasonalized time series of CRE along with the ENSO index. Following several previous studies (Deser \& Wallace 1990; Fu et al. 1996; Klein et al. 1999), the ENSO index is defined as the 5-month running mean of deseasonalized SST anomalies averaged over the tropical eastern Pacific (defined as the area between $5^{\circ} \mathrm{S}-5^{\circ} \mathrm{N}$ and the South American coast and $180^{\circ} \mathrm{W}$ ). The amplitude of interannual variation of $\mathrm{LW}$ broadband CRE is $\sim 0.5 \mathrm{Wm}^{-2}$. The running-mean time series of observed CRE and those of simulated by the AM2 and CanAM4 models are positively correlated with the ENSO index. They tend to have positive anomalies when the eastern Pacific SST anomalies are also positive (i.e., the El Niño state). GEOS-5 (red line in Fig. 8a) shows a different response to the ENSO index. The correlation between GEOS-5 broadband LW CRE time series and the ENSO index is slightly positive ( 0.02$)$ and but not statistically significant. The variation of each band CRE anomalies with the ENSO index closely follows the variation of LW broadband CRE anomalies, as shown in Fig. $8 \mathrm{~b}$ for Band1 (results from other bands not shown here).

When the 5-month running mean of the deseasonalized anomalies of $f_{C R E}$ is correlated with the ENSO index (Fig. 9), all three models agree with the observation on the signs of correlations over all bands excepts Band 4 (the ozone band). The correlation with the ENSO index is positive for Band 1 (the $\mathrm{H}_{2} \mathrm{O}$ band) and negative for Bands 3 and 5 (the window bands). Correlations using the observations tend to be smaller than those from the simulations, which is likely due to the more noisy nature of the observations. The correlations in Fig. 9 indicate to a 
large extent how the cloud top height varies at the interannual time scale with the ENSO index.

Both the observation and models suggest that, averaged over the entire tropical ocean, the mean infrared-effective cloud top averaged over the tropical oceans tends to be elevated when the ENSO index is positive (El Niño state) and vice-versa.

\section{Model intercomparisons and comparisons with observations: spatial distributions}

Discussions in the previous section were about the averages over the entire tropical oceans. In this section, spatial distributions of band-by-band CRE and $f_{C R E}$ are to be discussed. As explained in Section1, the map of absolute CRE of a particular band largely resembles the map of broadband LW CRE (right panels in Fig. 2) and highlights the contrast of absolute CRE between regions featuring high clouds (where broadband LW CRE is $\sim 60-80 \mathrm{Wm}^{-2}$ ) and regions dominated by low clouds (where broadband LW CRE is $\sim 10-20 \mathrm{Wm}^{-2}$ or even less). Therefore, in this section the focus is on the spatial distributions of $f_{\text {CRE. }}$. Because the broadband and band-byband CRE provide similar information, we mainly focus the discussion in this section to the spatial distributions of $f_{\text {CRE. }}$.

Figure 10a shows the observed Band1 $f_{C R E}$. As expected, the largest values of $f_{C R E}(\sim 0.25$ 0.35) are found over the regions with frequent occurrence of high clouds, such as ITCZ, SPCZ, and Indian Monsoon regions. The smallest values occur in regions frequently covered by marine stratus (i.e. low cloud), such as the Pacific coast off South America, the Namibia coast, and the ocean region west of Australia. Figures $10 \mathrm{~b}-10 \mathrm{~d}$ show the differences between modeled and observed Band $1 \mathrm{f}_{\mathrm{CRE}}$. Simulated climatological mean positions of ITCZ over the equatorial Pacific and Atlantic are south to the observed ones, and the widths of ITCZ are also different, which accounts for the negative differences around $8^{\circ} \mathrm{N}$ and the slight positive differences between 0- 
$10^{\circ} \mathrm{N}$ of the tropical Pacific and Atlantic. For regions where low clouds are prevalent, modeled $f_{C R E}$ are usually higher than the observed ones but the three models largely agree with each other.

For Band2 $\left(\mathrm{CO}_{2}\right.$ band, Fig. 10e), the contrast in $\mathrm{f}_{\mathrm{CRE}}$ between high-cloud and low-cloud regions ( 0.16 vs. 0.11$)$ is much smaller than that of Band1 ( 0.3 vs. 0.05$)$. The models largely disagree with each other (Fig. $10 \mathrm{f}-\mathrm{h}$ ) on the spatial distribution of $\mathrm{f}_{\mathrm{CRE}}$. Compared to the observation, AM2 considerably underestimates Band $2 \mathrm{f}_{\mathrm{CRE}}$ over the low-cloud regions but largely agrees with observations over the rest of tropical oceans. GEOS-5 uniformly overestimates Band2 $f_{\text {CRE }}$ everywhere over the tropical oceans by 0.02-0.04 with slightly larger overestimates for parts of the low-cloud regions. CanAM4 exceeds observations by 0.02-0.04 for the tropical oceans except the low-cloud region, where the difference is indeed slightly negative $(\sim-0.02$ to 0$)$.

The observed Band3 $f_{\text {CRE }}$ (Fig. 11a) peaks over the low-cloud regions and drops over the high-cloud regions. This is consistent with the variation of Band $3 f_{C R E}$ with cloud top pressure (e.g., Fig. 7a). Compared to the observation, the AM2 model overestimates Band $3 \mathrm{f}_{\mathrm{CRE}}$ nearly uniformly over the entire tropical oceans by $\sim 0.04$. The GEOS5 and CanAM4, on the other hand, underestimate Band $3 \mathrm{f}_{\mathrm{CRE}}$ over the low-cloud regions and are close to the observations for the rest regions of the domain with only slight positive or negative difference. These modelobservation differences are opposite to those for Band2, for which the GFDL AM2 underestimates over the low-cloud region and the GEOS5 and CanAM4 overestimate over majority of the tropical oceans. This is another way to expose the compensating biases among different bands in a GCM. As mentioned in Section 1 and Huang et al. (2010), such differences 
in modeled $f_{C R E}$ can be attributed to the differences in cloud top temperature. The differences between the AM2 and observations over both Band2 and Band3 consistently indicate that, on average, the cloud top temperature over the low-cloud regions are higher than observed ones, which leads to an overestimation of Band $3 f_{\text {CRE }}$ and the underestimation of Band $2 f_{\text {CRE. }}$. Similarly, for the GEOS-5, the mean cloud top temperature are lower than the observed ones, accounting for the underestimation of Band $3 f_{C R E}$ and the overestimation of Band $2 f_{C R E}$. Note it is known that there is still difficulty in simulating temperature profiles in the low-cloud regions (temperature inversion, boundary layer mixing, etc.). So difference between simulated and observed cloud top temperature could be due to cloud top height difference as well as boundary-layer temperature difference between models and observations. For the CanAM4, the overestimation for Band2 is generally smaller than in GEOS-5 over the low-cloud regions but still noticeable over certain areas, such as the Peru coast and Namibia coasts, which suggests lower cloud top temperature than observed ones in these regions.

The observed spatial map of Band4 $f_{C R E}$ (ozone band, Fig. 10e) is similar as that of Band3, owing to the fact that the ozone band is sensitive to the cloud and surface thermal contrast in a similar way as neighboring bands (i.e. Band3 and Band5). The ozone band in the AM2 model has a bandwidth $30 \mathrm{~cm}^{-1}$ shorter than that used in the observations and the GEOS-5 and CanAM4 models (Table 2), which accounts for the consistently smaller contribution in the AM2 (Fig. 10f) than in the observation. GEOS-5 agrees best with observations, even though smaller biases can still be seen over low-cloud regions consistent with those shown in Band3 over the same region. The calculated on-line ozone profiles continuously updated with the dynamical fields in the GEOS-5 simulation probably contribute to such good agreement. The spatial maps 
of Band5 $\mathrm{f}_{\mathrm{CRE}}$ and model-observation difference (not shown here) are similar to those of Band3 because the majority of the radiation of Band5 comes from the second window region (1100$\left.1200 \mathrm{~cm}^{-1}\right)$.

As mentioned in Section 2, the GFDL AM2 radiation scheme assumes all clouds to be non-scattering in the LW while the NASA GEOS-5 and CCCma CanAM4 explicitly take the scattering into account (though the treatments are different in GEOS-5 and CanAM4). Therefore, it is meaningful to estimate how much of the difference shown in Figs. 10 and 11 is due to the inclusion of scattering. Similar to Fig. 7, we use the same conceptual model to estimate such difference. In one case cloud is assumed to be non-scattering and, in another case, the scattering is solved by a 4-stream DISORT solver in the MODTRAN5 package (Berk et al., 2005). $f_{C R E}$ of the non-scattering case is shown in Fig. $12 \mathrm{a}$ and the difference between the two cases (scattering - non-scattering) is shown in Fig. 12b. For clouds below 10km, the difference is negligible except for Band3 and cloud top below $4 \mathrm{~km}$. For clouds above $10 \mathrm{~km}$, there are noticeable differences in all five bands. From $10-13 \mathrm{~km}$, the difference is within \pm 0.03 . The largest difference $(\sim-0.09)$ is seen in Band1 for cloud top height at $14 \mathrm{~km}$. Such large negative difference can be understood from two aspects: (1) according to the Wien's displacement law, for a cloud top height at $14 \mathrm{~km}$ (i.e., cloud top pressure at $\sim 150 \mathrm{mb}$ and cloud top temperature around $210 \mathrm{~K}$ ), the fractional contribution from Band1 is as large as that from Band3 (Fig. 12a); (2) for ice, the imaginary part of the index of refraction of has a minimum value at $410 \mathrm{~cm}^{-1}$ (Warren 1984; Warren \& Brandt 2008), which means the inclusion of scattering would lead to the strongest scattering effect around this wavenumber. Overall, Fig. $12 \mathrm{~b}$ indicates that inclusion or not of scattering in the radiation scheme should have minor or 
negligible impact on the $f_{C R E}$, unless the cloud top is very high $(\geq 14 \mathrm{~km})$. Note over the tropical oceans, only a small fraction clouds can have their tops at or above $14 \mathrm{~km}(\sim 150 \mathrm{hPa})$. Cloud penetrating radar observation suggests that this fraction is $10 \%$ or less and is concentrated in the core of ITCZ area (Haynes \& Stephens 2007). For the GCMs examined here, the monthly mean cloud fraction above $150 \mathrm{hPa}$ is about the same as the observed. Therefore, the discrepancies between models and observations for either averages or spatial distributions, are primarily due to the difference in the modeled and observed cloud properties themselves, and not due to the inclusion of scattering or the details of scattering treatment in the radiation scheme.

\section{Conclusion and discussion}

Taking advantage of a multi-year multi-band LW CRE dataset over the tropical oceans derived from collocated AIRS and CERES observations, this study evaluates in details the LW band-by-band CRE simulated by three different GCMs. It quantitatively demonstrates that, even when the model-simulated broadband LW CRE largely agrees with the observed one, compensating biases from different LW bands can be prominent. The band-by-band CRE differences between models and observations can be as large as the difference in the LW broadband CRE. We prefer to use in our analysis the fractional contribution of each band to the LW CRE, $f_{C R E}$, which has the unique advantage of being sensitive to cloud top height/temperature and not sensitive to cloud fraction. This property of $f_{C R E}$ enables us to separate the contribution of cloud fraction and cloud top height to the top-of-atmosphere LW flux. The averaged $f_{C R E}$ over the tropical oceans, their seasonal cycles, and their interannual variations are also examined. Band $1 f_{C R E}\left(H_{2} \mathrm{O}\right.$ band) is strongly anti-correlated with Band3 $f_{C R E}$ 
and Band5 $f_{C R E}$ (window regions). The observed seasonal cycle is consistent with the seasonal cycles of ISCCP cloud top pressure climatology on both the phase and the amplitude. The simulated seasonal cycles of $f_{C R E}$ agree with observations on the phase but not on the amplitude which is much smaller in the simulatons, indicating smaller seasonal variations of simulated cloud top pressure, on average. As for interannual variations, observations and all GCMs agree on the positive (negative) correlations between Band1 $f_{C R E}$ (Band3 and Band5 $f_{C R E}$ ) and the ENSO index. Spatial distributions of band-by-band $f_{\text {CRE }}$ highlight the large discrepancies between models and observations in the regions with frequent occurrence of low clouds. While the total LW CRE is usually not used for the study of low clouds, fractional CRE contributions are sensitive to the low cloud top height (or equivalently, low cloud temperature). This complements the diagnostics of low cloud with shortwave reflectance, which is more sensitive to cloud liquid path than to the cloud top height/temperature. Cloud top height only directly affects the shortwave reflectance by changing the amount of solar absorption due to water vapor above the cloud. But change of cloud top height dominates the change of LW band-byband $f_{C R E}$. Therefore, such band-by-band $f_{C R E}$ offers a useful diagnostics for the low clouds simulated by the models.

While this analysis focuses on $\mathrm{f}_{\mathrm{CRE}}$ for the reason articulated in Section 1, both absolute LW CRE and $f_{C R E}$ of each band are important to diagnose the quality of cloud simulations. Cloud fraction significantly affects the absolute LW CRE while $f_{C R E}$ is more sensitive to cloud top height/temperature. If a model is poor in producing correct cloud fractions for a certain type of cloud (e.g., the underestimation of low cloud fractions in the GEOS-5 model as noted in comparison with observed low cloud fractions, ), it would be logical and practical to first 
address the issue of cloud fraction and then evaluate the $f_{\text {CRE. }}$. For both model evaluation and cloud feedback study, $f_{C R E}$ is a meaningful metric to use and it complements the absolute broadband LW CRE.

To our knowledge, this is the first study to compare the simulated band-by-band LW CRE from multiple GCMs. The good agreement between NASA GEOS-5 and observation in the ozone-band CRE (Fig. 11) suggests that, when the detailed band-by-band decomposition of LW CRE are scrutinized against observations, the effect of ozone cannot be neglected. This has implication for the tuning of GCMs to reach the TOA energy balance as well as the climate projections in the presence of ozone recovery. Generally speaking, it is possible, if not very likely, to tune GCM to achieve a the TOA balance between outgoing longwave flux and net incoming shortwave flux for current climate while the band-by-band decomposition of LW flux (or LW CRE) is different from that observed. For example, the bias in the TOA energy imbalance due to simple representation of time-varying ozone fields can be "tuned" away by adjusting some less-constrained parameters in various GCM parameterization schemes. What is unknown, however, is what the consequence would be for future climate projection simulated by the same GCM when such incorrect band-by-band composition of TOA flux for the current climate exists. Our future work will try to address this kind of question by analyzing the band-by-band flux and CRE from simulations carried out for different future scenarios prescribed by the IPCC assessment.

\section{Acknowledgements:}

We wish to thank H.W. Chuang for her assistance in data processing at the early stage of this work. The lead author is greatly indebted to NOAA GFDL for the generosity of providing 
computing resources for the GFDL AM2 simulation. The AIRS data were obtained from NASA GSFC DAAC and the CERES data from NASA Langley DAAC. This research is supported by NSF AGS CLD program under grant NSF ATM 0755310 and NASA Terra/Aqua program under grant NNX11AH55G awarded to the University of Michigan. L. Oreopoulos acknowledges funding support from NASA's Modeling Analysis and Prediction program managed by D. Considine.

\section{References:}

Allan, R. P., and M. A. Ringer, 2003: Inconsistencies between satellite estimates of longwave cloud forcing and dynamical fields from reanalyses. Geophys. Res. Lett., 30(9), 1491, doi: 10.1029/2003GL017019.

Allan, R. P., M. A. Ringer, J. A. Pamment, and A. Slingo, 2004: Simulation of the Earth's radiation budget by the European Centre for Medium-Range Weather Forecasts 40-year reanalysis (ERA40). J. Geophys. Res., 109, D18107, doi:10.1029/2004JD004816.

Berk, A., et al., 2005: MODTRAN5: A reformulated atmospheric band model with auxiliary species and practical multiple scattering options, Proc. SPIE Int. Soc. Opt. Eng., 5655, 88.

Chou, M.-D., M. J. Suarez, X.-Z. Liang, and Michael M. H-Yan, 2003: A Thermal Infrared Radiation Parameterization for Atmospheric Studies. Technical Report Series on Global Modeling and Data Assimilation, NASA/TM-2001-104606, Vol. 19, 65 pp.

Cionni, I., et al., 2011: Ozone database in support of CMIP5 simulations: Results and corresponding radiative forcing, Atmos. Chem. Phys., 11, 11267-11292, doi:10.5194/acp11-11267-2011. 
Deser, C., and J. M. Wallace, 1990: Large-scale atmospheric circulation features of warm and cold episodes in the tropical Pacific. J. Climate, 3, 1254-1281.

Fu, R., W. T. Liu, and R. E. Dickinson, 1996: Response of tropical clouds to the interannual variation of sear surface temperature. J. Climate, 9, 616-634.

GFDL GAMDT (Global Atmosphere Model Development Team), 2004: The new GFDL global atmosphere and land model AM2_LM2: Evaluation with prescribed SST simulations. J. Climate, 17, 4641-4673.

Haynes, J. M., and G. L. Stephens, 2007: Tropical oceanic cloudiness and the incidence of precipitation: Early results from CloudSat. Geophys. Res. Lett., 34, L09811, doi:10.1029/2007GL029335.

Huang, X. L., V. Ramaswamy, and M. D. Schwarzkopf, 2006: Quantification of the source of errors in AM2 simulated tropical clear-sky outgoing longwave radiation. J. Geophys. Res.Atmos., 111, D14107, doi:10.1029/2005JD006576.

-, W. Z. Yang, N. G. Loeb, and V. Ramasawamy, 2008: Spectrally resolved fluxes derived from collocated AIRS and CERES measurements and their application in model evaluation: clear sky over the tropical oceans, J. Geophys. Res.-Atmos., 113, D09110, doi: 10.1029/2007JD009219.

-, N. G. Loeb, and W. Z. Yang, 2010: Spectrally resolved fluxes derived from collocated AIRS and CERES measurements and their application in model evaluation:2. cloudy sky and band-by-band cloud radiative forcing over the tropical oceans, J. Geophys. Res.-Atmos., 115, D21101,doi:10.1029/2010JD013932. 
Huang, Y., V. Ramaswamy, X. L. Huang, Q. Fu, and C. Bardeen, 2007: A strict test in climate modeling with spectrally revolved radiances: GCM simulation versus AIRS observations, Geophys. Res. Lett., 34, L24707, doi:10.1029/2007GL031409.

Klein, Stephen A., Brain J. Soden, and Ngar-Cheung Lau, 1999: Remote sea surface temperature variations during ENSO: evidence for a tropical atmospheric bridge. J. Climate, 12, 917-932.

Li, J., 2002, Accounting for Unresolved Clouds in a 1D Infrared Radiative Transfer Model. Part I: Solution for Radiative Transfer, Including Cloud Scattering and Overlap. J. Atmos. Sci., 59, 3302-3320.

Li, J., and H. W. Barker, 2005: A radiation algorithm with correlated-k distribution. Part I: local thermal equilibrium. J. Atmos. Sci., 62, 286-309.

Li. J. and Q. Fu, 2000: Absorption approximation with scattering effect for infrared radiation. J. Atmos. Sci., 57, $2905-2914$.

McClatchy, R. A. et al., 1972: Optical Properties of the Atmosphere, Third Edition, Air Force Geophysical Laboratory Technical Report, AFCRL-72-0497, 80 pp.

Molod, A., L. Takacs, M. Suarez, J. Bacmeister, I-S. Song, A. Eichmann and Y. Chang, 2011: The GEOS-5 Atmospheric General Circulation Model: Mean Climate and Development from MERRA to Fortuna. NASA Technical Report Series on Global Modeling and Data Assimilation, NASA/TM-2008-104606, Vol. 28, 112 pp.

Paul,J., F.Fortuin, and H. Kelder, 1998: An ozone climatology based on ozonesonde and satellite measurements. J. Geophys. Res., 103(D24), 31,709-31,734. 
Pincus, R., H. W. Barker and J.-J. Morcrette, 2003: A fast, flexible, approximate technique for computing radiative transfer in inhomogeneous cloud fields. J. Geophys. Res., 108(D13), 4376.

Ramanathan, V., R. D. Cess, E. F. Harrison, P. Minnis, B. R. Barkstrom, E. Ahmad and D. Hartmann, 1989: Cloud-Radiative Forcing and Climate-Results from the Earth Radiation Budget Experiment. Science, 243, 57-63.

Raval, A., A. H. Oort, and V. Ramaswamy, 1994: Observed dependence of outgoing longwave radiation on sea surface temperature and moisture. J. Climate, 7, 807-821.

Rienecker M. M, M. J. Suarez, R. Todling, J. Bacmeister, L. Takacs, H.-C. Liu, W. Gu, M. Sienkiewicz, R. D. Koster, R. Gelaro, I. Stajner, and J. E. Nielsen, 2008: The GEOS-5 Data Assimilation System - Documentation of Versions 5.0.1, 5.1.0, and 5.2.0. NASA/TM-2008104606, Vol. 27, 118 pp.

Rossow, W. B., and R. A., Schiffer, 1991: ISCCP cloud data products. Bull. Amer. Meteor. Soc., 72, 2-20.

Rossow, W. B., and R. A., Schiffer, 1999: Advances in understanding clouds from ISCCP. Bull. Amer. Meteor. Soc., 80, 2261-2288.

Schwarzkopf, M. D., and V. Ramaswamy, 1999: Radiative effects of $\mathrm{CH}_{4}, \mathrm{~N}_{2} \mathrm{O}$, halocarbons and the foreign-broadened $\mathrm{H}_{2} \mathrm{O}$ continuum: A GCM experiment. J. Geophys. Res., 104(D8), 9467-9488, doi:10.1029/1999JD900003. 
Slingo, A., J. A. Pamment, and M. J. Webb, 1998: A 15-year simulation of the clear-sky greenhouse effect using the ECMWF reanalyses: Fluxes and comparisons with ERBE. J. Climate, 11, 690-708.

von Salzen, K., J. Scinocca, N. McFarlane, J. Li, J. Cole, D. Plummer, C. Reader, X. Ma, M. Lazare, and L. Solheim, 2012: The Canadian Fourth Generation Atmospheric Global Climate Model (CanAM4): Simulation of Clouds and Precipitation and Their Responses to Short-Term Climate Variability. Atmosphere-Ocean, Submitted.

Warren, S. G., 1984: Optical constants of ice from the ultraviolet to the microwave. Applied Optics, 23, 1206-1225.

-, and R. E. Brandt, 2008: Optical constants of ice from the ultraviolet to the microwave: A revised compilation. J. Geophys. Res., 113, D14220, doi:10.1029/2007JD009744.

Wielicki, B. A., et al., 2002: Evidence for large decadal variability in the tropical mean radiative energy budget. Science, $295,841-844$.

Wong, T., et al., 2006: Reexamination of the observed decadal variability of the earth radiation budget using altitude-corrected ERBE/ERBS nonscanner WFOV data. J. Climate, 19, 40284040.

Yang, S. K., Y. T. Hou, A. J. Miller, and K. A. Campana, 1999: Evaluation of the earth radiation budget in NCEP-NCAR reanalysis with ERBE. J. Climate, 12, 477-493. 


\section{Tables}

Table 1. Difference between OLR estimated from AIRS spectra with algorithms described in

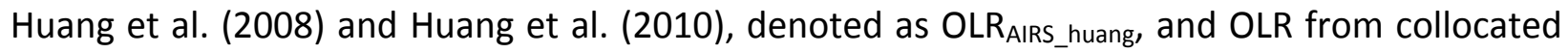
CERES measurements, denoted as OLR here. Number of collocated observations as well as the mean difference and standard deviation are listed for each year as mean \pm standard deviation.

\begin{tabular}{|c|c|c|c|c|}
\hline & \multicolumn{2}{|c|}{ Clear sky over the ocean } & \multicolumn{2}{c|}{ Cloudy sky over the ocean } \\
\hline & $\begin{array}{c}\text { Number of } \\
\text { observations }\end{array}$ & $\begin{array}{c}\text { OLR }_{\text {AIRS_huang }}-\text { OLR } \\
\left(\mathrm{Wm}_{\text {CERES }}\right)^{-2}\end{array}$ & $\begin{array}{c}\text { Number of } \\
\text { observations }\end{array}$ & $\begin{array}{c}\text { OLR }_{\text {AIRS_huang }}-\text { OLR }_{\text {CERES }} \\
\left(\mathrm{Wm}^{-2}\right)\end{array}$ \\
\hline $\mathbf{2 0 0 3}$ & $1.05 \times 10^{6}$ & $0.84 \pm 1.56$ & $1.18 \times 10^{7}$ & $2.66 \pm 5.68$ \\
\hline $\mathbf{2 0 0 4}$ & $1.08 \times 10^{6}$ & $0.67 \pm 1.52$ & $1.24 \times 10^{7}$ & $2.15 \pm 5.51$ \\
\hline $\mathbf{2 0 0 5}$ & $1.08 \times 10^{6}$ & $1.42 \pm 1.68$ & $1.26 \times 10^{7}$ & $2.88 \pm 5.79$ \\
\hline $\mathbf{2 0 0 6}$ & $1.08 \times 10^{6}$ & $1.55 \pm 1.74$ & $1.27 \times 10^{7}$ & $2.94 \pm 5.92$ \\
\hline $\mathbf{2 0 0 7}$ & $1.04 \times 10^{6}$ & $1.73 \pm 1.86$ & $1.27 \times 10^{7}$ & $3.00 \pm 5.92$ \\
\hline
\end{tabular}

Table 2. The LW bands used in each GCM as well as the five bands used in this study for the comparisons across all GCMs and observations. The bandwidths are given in wavenumber $\left(\mathrm{cm}^{-}\right.$ $\left.{ }^{1}\right)$. The major absorbers of each band are also listed.

\begin{tabular}{|c|c|c|c|c|}
\hline $\begin{array}{l}\text { Major } \\
\text { absorber(s) }\end{array}$ & $\begin{array}{l}\text { Band used in this } \\
\text { study }\left(\mathrm{cm}^{-1}\right)\end{array}$ & GFDL Band ID & GEOS-5 Band ID & CanAM4 Band ID \\
\hline $\mathrm{H}_{2} \mathrm{O}$ & $\begin{array}{l}\text { Band1: } 0-560 \&> \\
1400\end{array}$ & $\begin{array}{l}\text { Bands } 1 \& 8 \\
(0-560 \&>1400)\end{array}$ & $\begin{array}{l}\text { Bands1-2 (0-540) \& } \\
\text { Bands 8-9 (>1380) }\end{array}$ & $\begin{array}{l}\text { Bands 1-3 }(>1400) \\
\text { Bands 8-9 }(0-540)\end{array}$ \\
\hline $\mathrm{CO}_{2}$ & Band2: $560-800$ & $\begin{array}{l}\text { Band } 2 \\
(560-800)\end{array}$ & $\begin{array}{l}\text { Bands 3\&10 } \\
(540-800)\end{array}$ & Band $7(540-800)$ \\
\hline $\begin{array}{l}\mathrm{H} 2 \mathrm{O} \\
\text { continuum }\end{array}$ & Band3: $800-980$ & $\begin{array}{l}\text { Band 3-4 } \\
(800-990)\end{array}$ & $\begin{array}{l}\text { Band4 } \\
(800-980)\end{array}$ & Band 6 (800-980) \\
\hline $\mathrm{O}_{3}$ & Band4: 980-1100 & $\begin{array}{l}\text { Band 5 } \\
(990-1070)\end{array}$ & $\begin{array}{l}\text { Band5 } \\
(980-1100)\end{array}$ & Band 5 (980-1100) \\
\hline $\begin{array}{l}\mathrm{H} 2 \mathrm{O} \\
\text { continuum; } \\
\mathrm{CH}_{4}, \mathrm{~N}_{2} \mathrm{O}\end{array}$ & Band5: $1100-1400$ & $\begin{array}{l}\text { Band } 6(1070-1200) \\
\text { Band } 7(1200-1400)\end{array}$ & $\begin{array}{l}\text { Band6 (1100-1215) } \\
\text { Band7 (1215-1380) }\end{array}$ & Band 4 (1100-1400) \\
\hline
\end{tabular}


Table 3. Multi-year means of LW broadband and band-by-band CRE averaged over the tropical oceans. The numbers in parentheses are the fractional contribution to the total LW broadband CRE $\left(f_{C R E}\right)$. The $A M 2$ results for Band4 are shaded and in italics to denote the prominent bandwidth differences between the GFDL AM2 model and the other two GCMs and observations (refer to Section 2 for detailed discussions).

\begin{tabular}{|c|c|c|c|c|}
\hline & Observation & GFDL AM2 & NASA GEOS-5 & CCCma CanAM4 \\
& $\left(\mathrm{Wm}^{-2}\right)$ & $\left(\mathrm{Wm}^{-2}\right)$ & $\left(\mathrm{Wm}^{-2}\right)$ & $\left(\mathrm{Wm}^{-2}\right)$ \\
\hline LW broadband & $28.5(100 \%)$ & $28.0(100 \%)$ & $28.3(100 \%)$ & $27.3(100 \%)$ \\
\hline $\begin{array}{c}\text { Band1: 0-560 \& }>1400 \\
\mathrm{~cm}^{-1}\end{array}$ & $5.59(19.6 \%)$ & $5.31(19.0 \%)$ & $5.11(18.1 \%)$ & $4.47(16.3 \%)$ \\
\hline Band2: $560-800 \mathrm{~cm}^{-1}$ & $4.33(15.2 \%)$ & $3.72(13.3 \%)$ & $5.07(18.0 \%)$ & $4.82(17.6 \%)$ \\
\hline Band3: $800-980 \mathrm{~cm}^{-1}$ & $9.25(32.4 \%)$ & $9.96(35.6 \%)$ & $9.03(32.0 \%)$ & $8.80(32.2 \%)$ \\
\hline Band4: $980-1100 \mathrm{~cm}^{-1}$ & $3.72(13.0 \%)$ & $1.68(6.0 \%)$ & $3.63(12.9 \%)$ & $3.75(13.7 \%)$ \\
\hline Band5: $1100-1400 \mathrm{~cm}^{-1}$ & $5.62(19.7 \%)$ & $7.32(26.2 \%)$ & $5.41(19.2 \%)$ & $5.50(20.1 \%)$ \\
\hline
\end{tabular}




\section{Figure Captions}

Figure 1. (a) The percentage flux difference due to differences between intrinsic GCM bandwidths defined in each GCM and the bandwidths used in this study (refer to Table 2 for the details). Flux is calculated for a blackbody at 220K. (b) Same as (a), but for a blackbody at 298K.

(c) The flux difference between (a) and (b) expressed in percentage.

Figure 2. The multi-year mean of all-sky OLR (left panels) and LW broadband LW CRE (right panels) derived from collocated AIRS and CERES observations, the GFDL AM2, NASA GEOS5, and CCCma CanAM4 model simulations. Note that color scales are different for the left panel and the right panels.

Figure 3. The mean seasonal cycles of Band1 CRE (blue lines), Band2 CRE (green lines), and Band3 CRE(red lines). Definitions of bandwidths can be found in Table 2. For better visualization, CREs from the observation and GEOS-5 model are plotted on one scale $\left( \pm 1 \mathrm{Wm}^{-2}\right)$ and those from the AM2 and CanAM4 model are plotted on another scale $\left( \pm 0.5 \mathrm{Wm}^{-2}\right)$.

Figure 4. The mean seasonal cycle of $f_{C R E}$, the fractional contribution of a band to the total LW CRE. Band 1-3 are plotted in blue lines, green lines, and red lines, respectively.

Figure 5. Correlation coefficients between the mean seasonal cycle of Band1 and that of all other bands. The observed relation is plotted in blue dotted line. The GFDM AM2, NASA GEOS-5, and CanAM4 models are plotted in green, red, and cyan dotted lines, respectively.

Figure 6. (a) The ISCCP climatological seasonal cycle (deviations from the climatological mean) of cloud fraction for high cloud (blue), middle cloud (green), and low cloud (red) averaged over 
the tropical oceans. (b) The ISCCP climatological seasonal cycle of cloud top pressure averaged over the tropical oceans.

Figure 7. (a) Change of Band $1 f_{C R E}$ and Band $3 f_{C R E}$ with respect to the cloud top pressure in a simple conceptual model in which cloud is assumed to be optically thick and typical tropical sounding profiles are used. Radiative calculation is done with MODTRAN5. (b) Observed standard deviations of the seasonal cycles of Band1 $f_{C R E}$ and Band3 $f_{C R E}$ vs. estimated counterparts. Black dash line denotes the 1:1 slope.

Figure 8. (a) 5-month running means of deseasonalized anomalies of total LW CRE from the observations (blue line), the GFDL AM2 (green line), NASA GEOS-5 output (red line), and CCCma CanAM4 (cyan line) simulations. The ENSO index is plotted in gray dash line. (b) Same as (a) except for the Band1 CRE instead of total LW CRE. Following previous studies, the ENSO index is defined as 5-month running mean of the SST anomalies averaged over the region of $5^{\circ} \mathrm{S}-5^{\circ} \mathrm{N}$, the South American coast and $180^{\circ} \mathrm{W}$.

Figure 9. Correlation coefficients between the ENSO index and the 5-month running means of deseasonalized $\mathrm{f}_{\mathrm{CRE}}$ of each band. Results from observation and three models are shown in different colors. Figure 10 (a) Map of observed long-term mean of Band1 $f_{\text {CRE. }}$ (b)-(d) Maps of model-observation difference in the long-term mean of Band1 $f_{C R E}$ for the GFDL AM2, NASA GEOS-5, and CCCma CanAM4 simulations, respectively. Note the same color scale is used for (b)-(d). (e) same as (a) except for Band2 $\mathrm{f}_{\text {CRE. }}$ (f)-(h) same as (b)-(d) except for Band2 $\mathrm{f}_{\mathrm{CRE}}$. 
Figure 11. Same as Figure 10 except for Band3 $f_{C R E}$ and Band4 $f_{C R E}$.

Figure 12. (a) Band-by-band $f_{C R E}$ for different cloud top height $(2 \mathrm{~km}-14 \mathrm{~km})$. Clouds are assumed to be opaque and non-scattering. (b) The difference in $f_{C R E}$ between the case of scattering cloud and the case of non-scattering cloud when everything else remains the same. 


\section{$\mathrm{T}=220 \mathrm{~K}$}

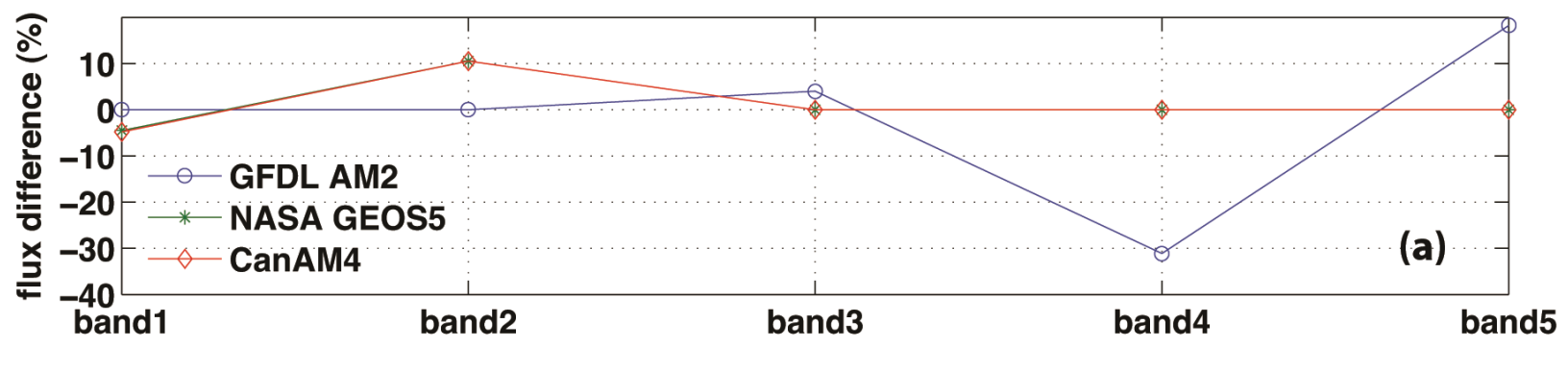

Flux difference (298K - 220K)

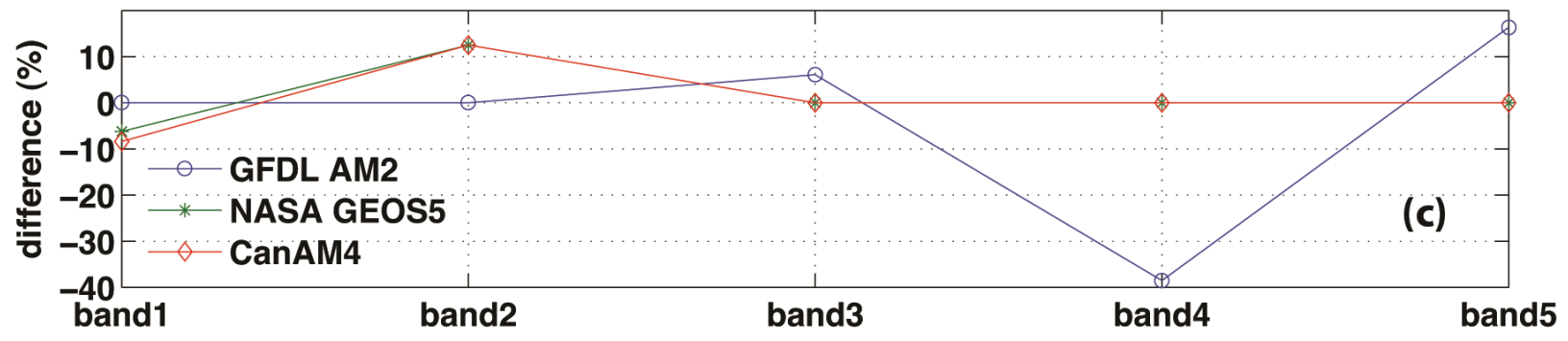

Figure 1. (a) The percentage flux difference due to differences between intrinsic GCM bandwidths defined in each GCM and the bandwidths used in this study (refer to Table 2 for the details). Flux is calculated for a blackbody at 220K. (b) Same as (a), but for a blackbody at 298K.

(c) The flux difference between (a) and (b) expressed in percentage. 

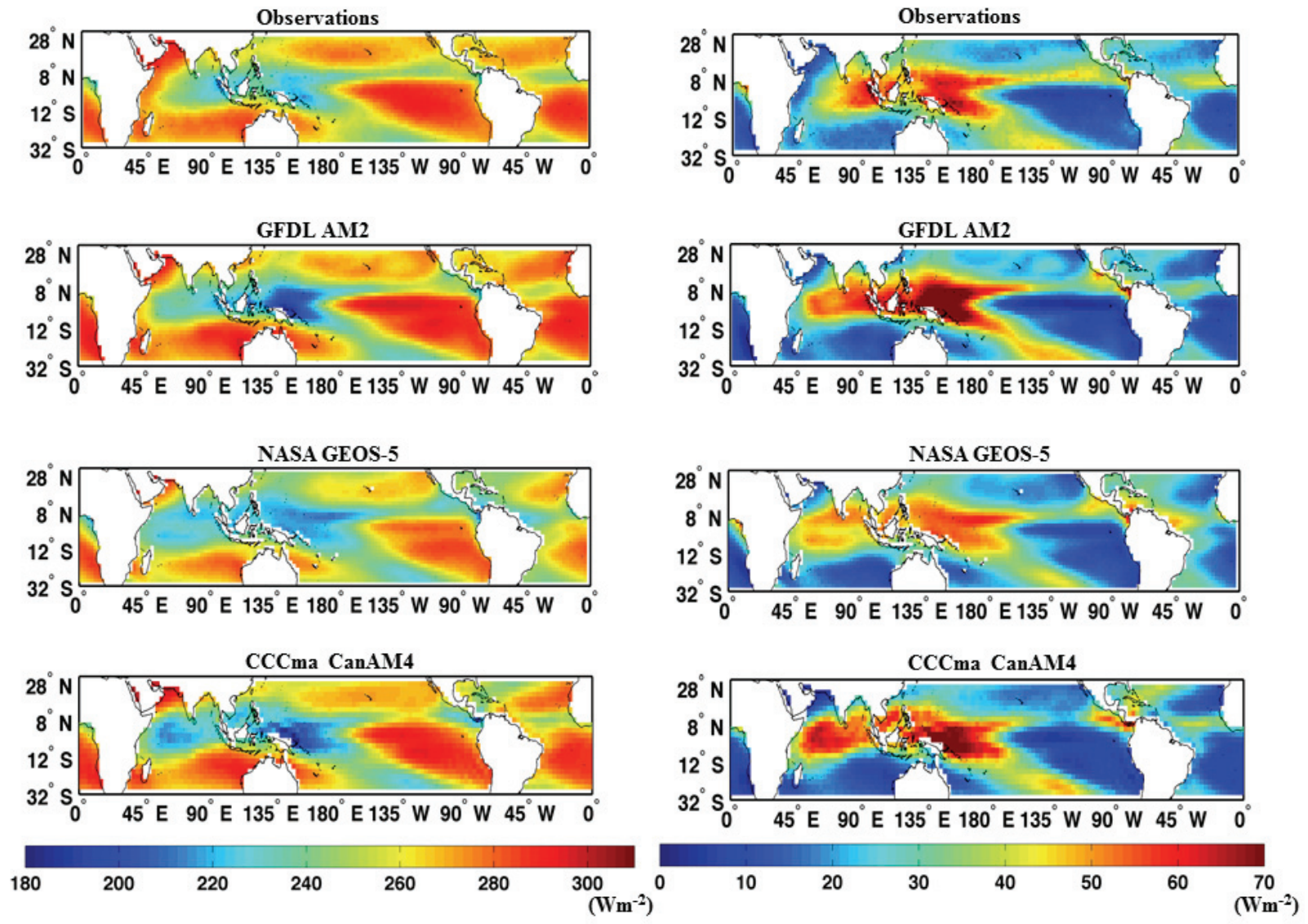

Figure 2. The multi-year mean of all-sky OLR (left panels) and LW broadband LW CRE (right panels) derived from collocated AIRS and CERES observations, the GFDL AM2, NASA GEOS5, and CCCma CanAM4 model simulations. Note that color scales are different for the left panel and the right panels. 

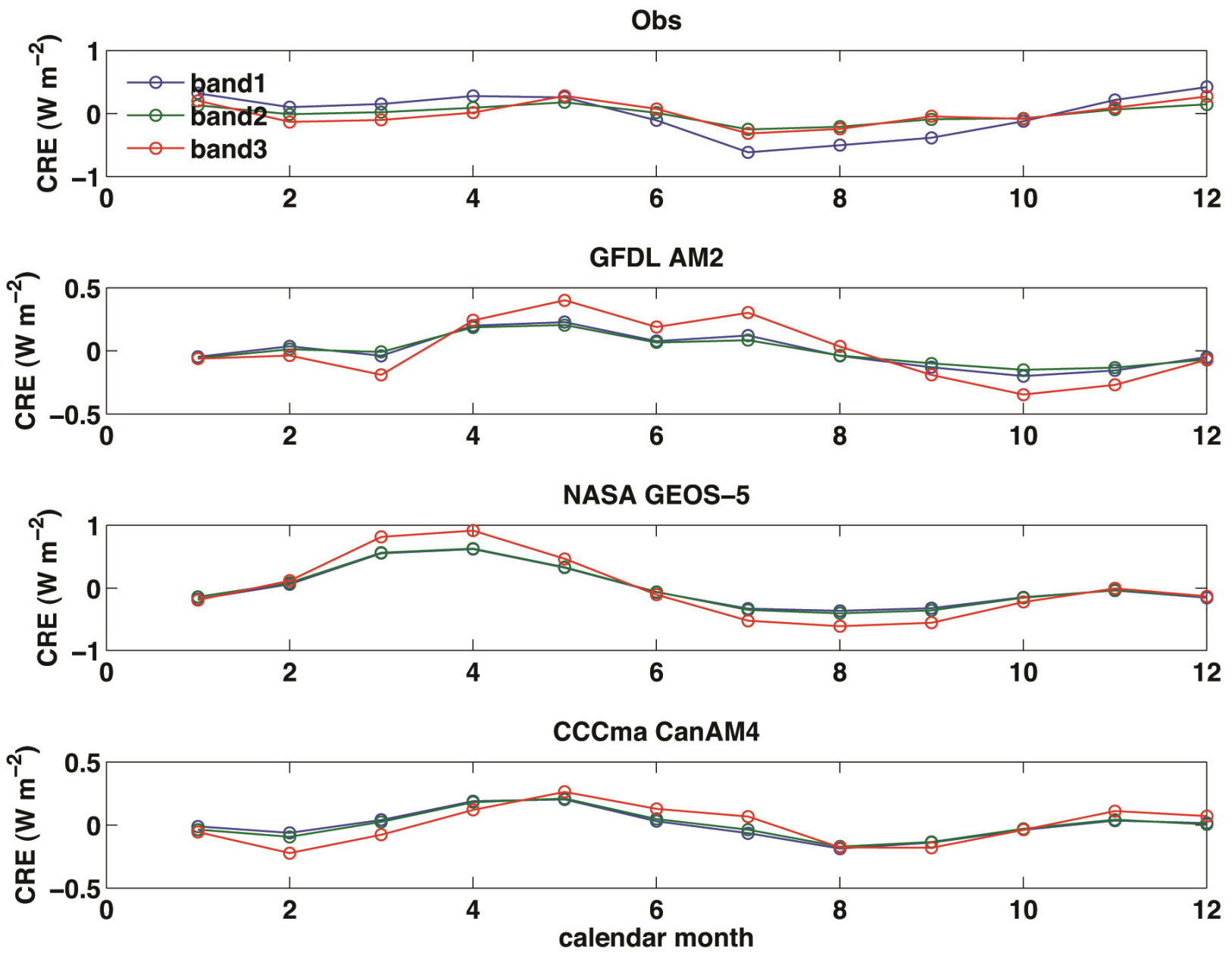

Figure 3. The mean seasonal cycles of Band1 CRE (blue lines), Band2 CRE (green lines), and Band3 CRE(red lines). Definitions of bandwidths can be found in Table 2. For better visualization, CREs from the observation and GEOS-5 model are plotted on one scale $\left( \pm 1 \mathrm{Wm}^{-2}\right)$ and those from the AM2 and CanAM4 model are plotted on another scale $\left( \pm 0.5 \mathrm{Wm}^{-2}\right)$. 


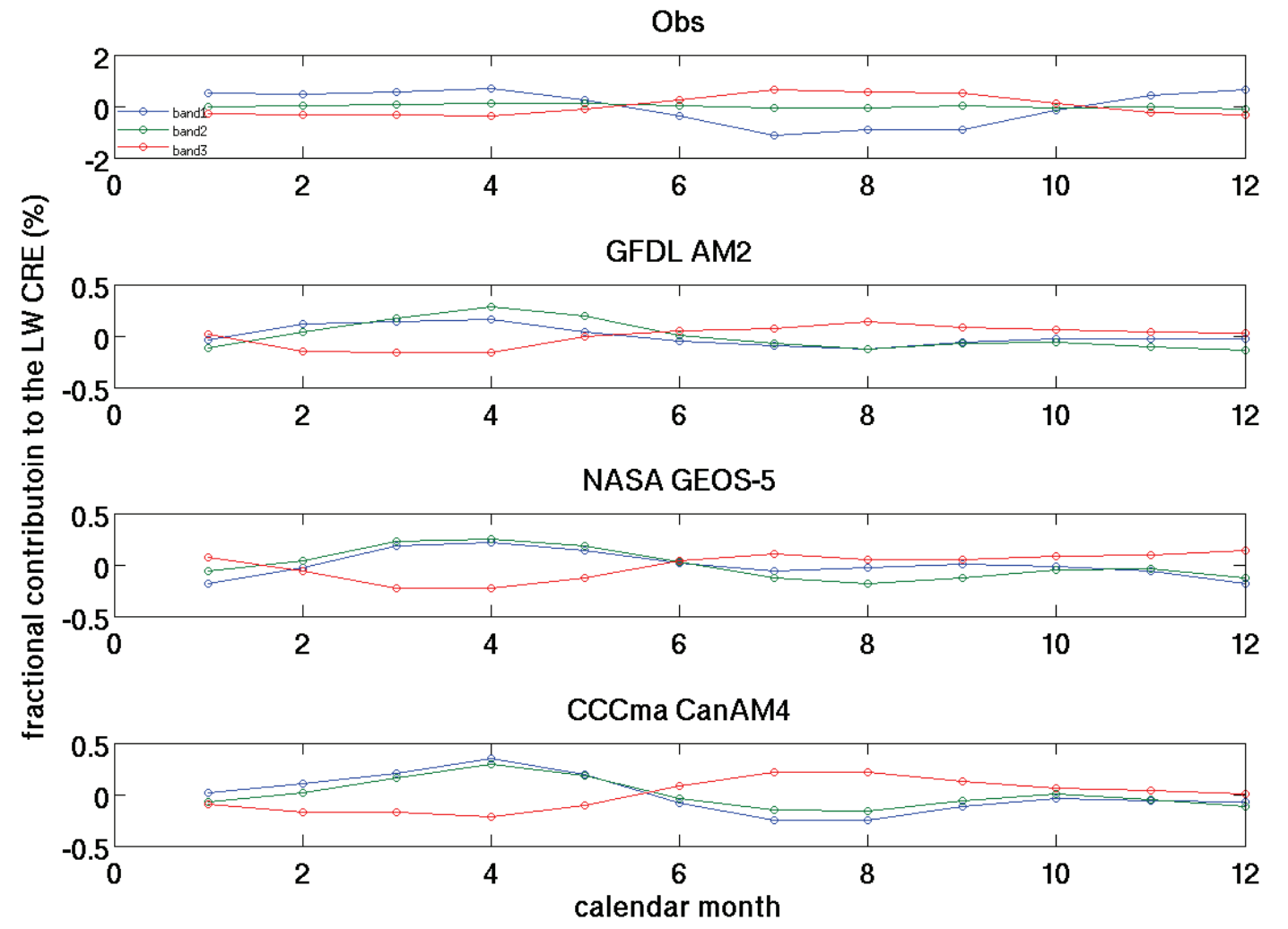

Figure 4. The mean seasonal cycle of $f_{C R E}$, the fractional contribution of a band to the total $L W$ CRE. Band 1-3 are plotted in blue lines, green lines, and red lines, respectively. 


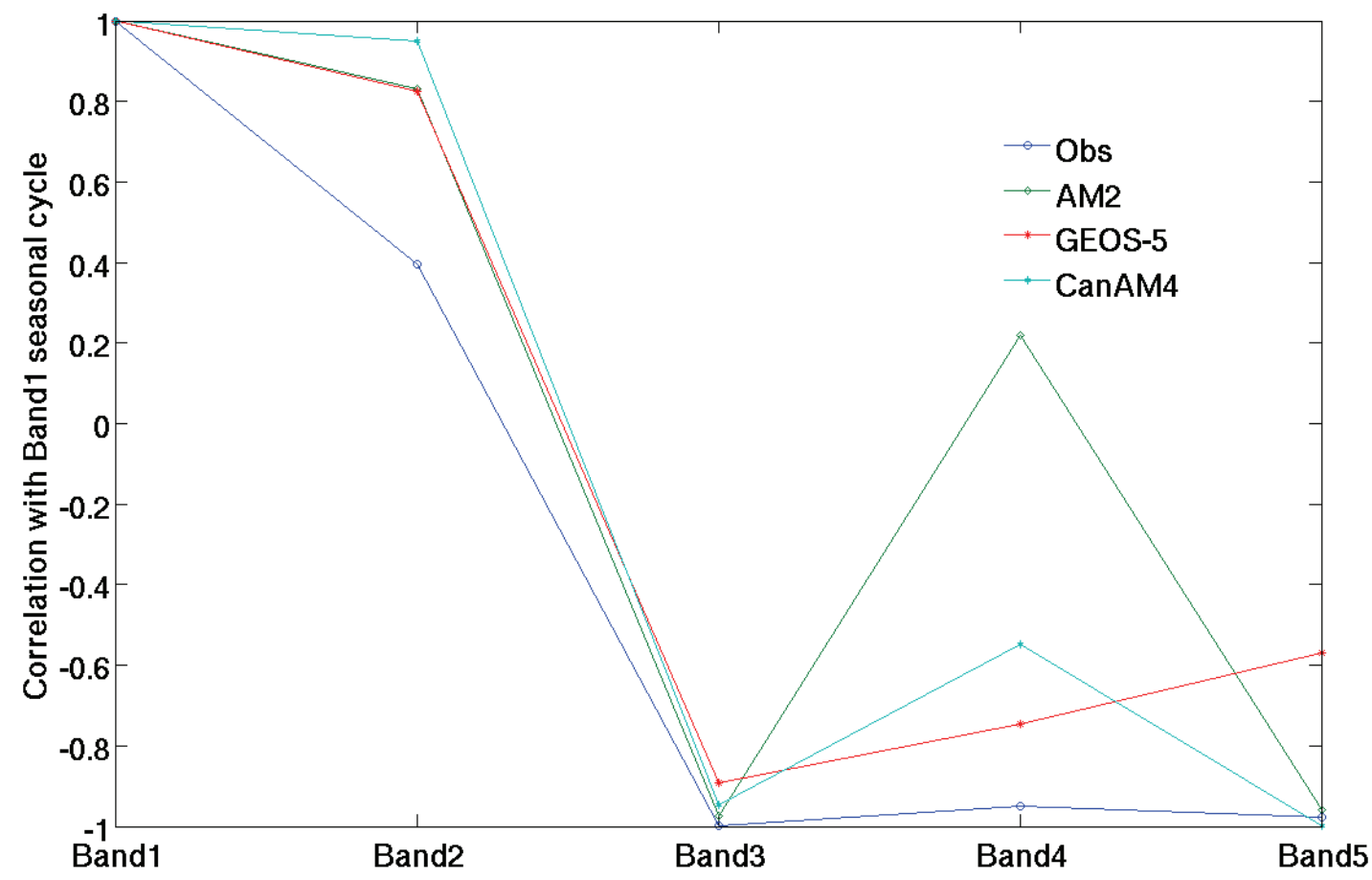

Figure 5. Correlation coefficients between the mean seasonal cycle of Band1 and that of all other bands. The observed relation is plotted in blue dotted line. The GFDM AM2, NASA GEOS-5, and CanAM4 models are plotted in green, red, and cyan dotted lines, respectively. 

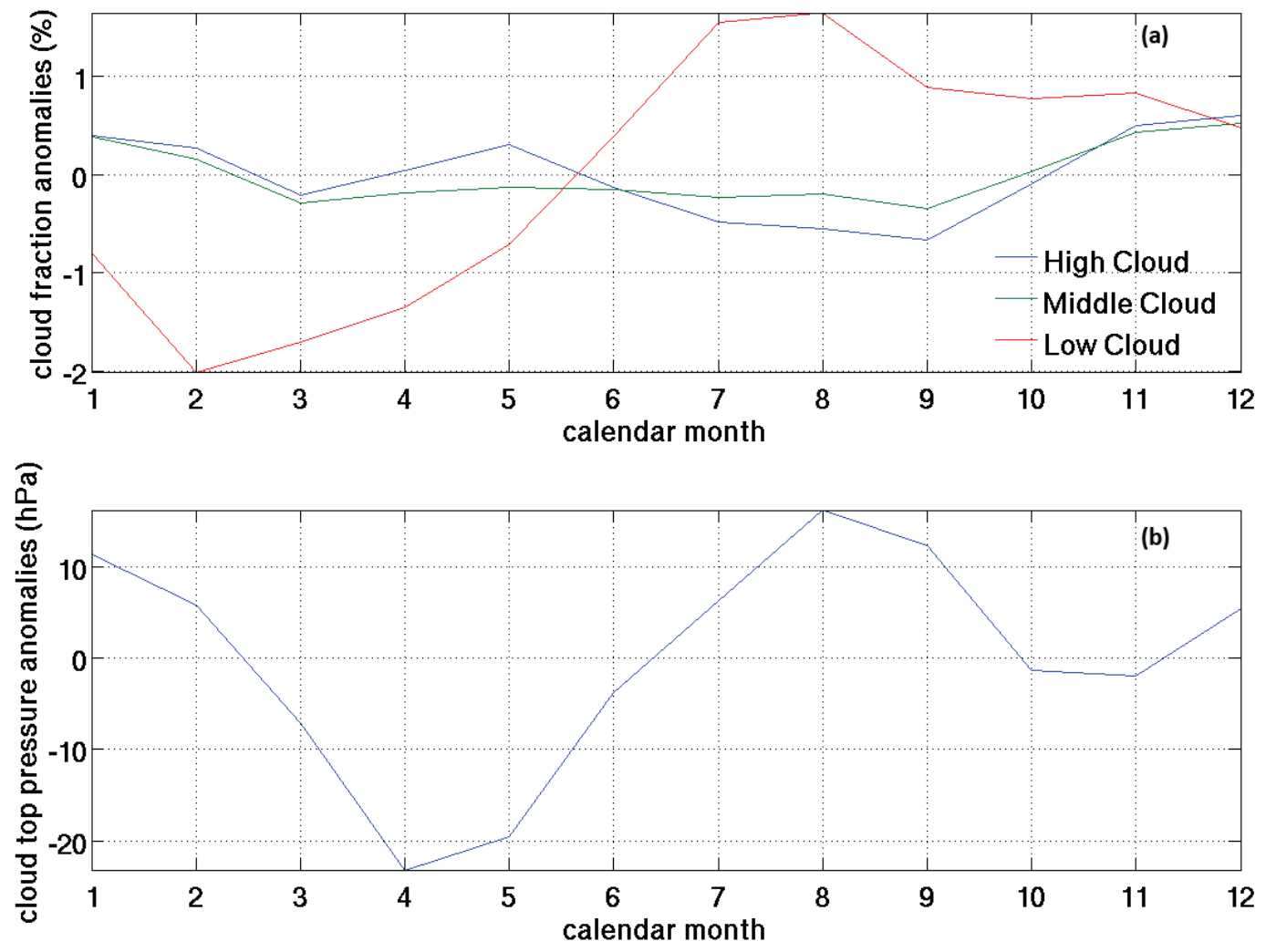

Figure 6. (a) The ISCCP climatological seasonal cycle (deviations from the climatological mean) of cloud fraction for high cloud (blue), middle cloud (green), and low cloud (red) averaged over the tropical oceans. (b) The ISCCP climatological seasonal cycle of cloud top pressure averaged over the tropical oceans. 

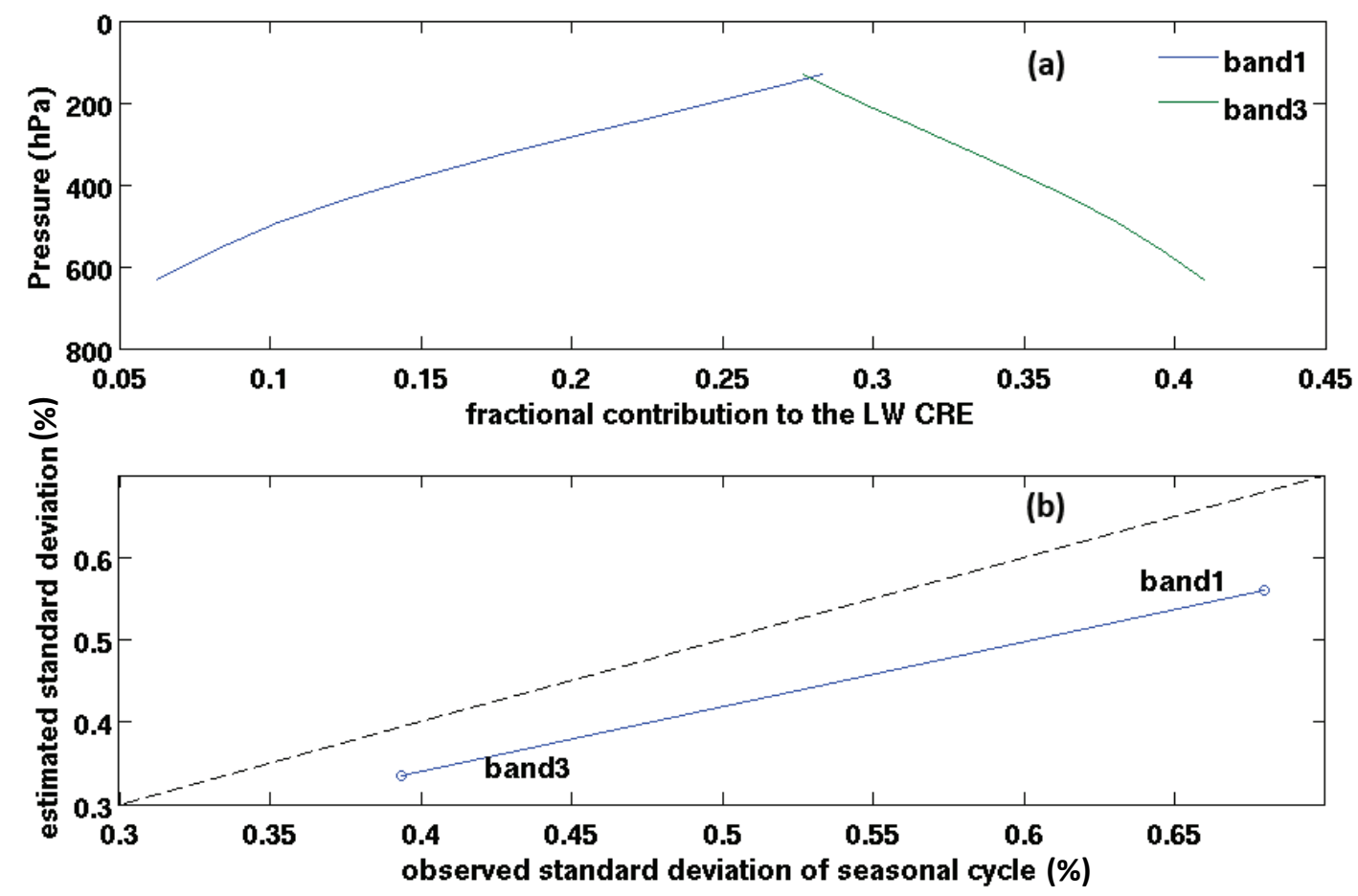

Figure 7. (a) Change of Band1 $f_{C R E}$ and Band $3 f_{C R E}$ with respect to the cloud top pressure in a simple conceptual model in which cloud is assumed to be optically thick and typical tropical sounding profiles are used. Radiative calculation is done with MODTRAN5. (b) Observed standard deviations of the seasonal cycles of Band $1 f_{C R E}$ and Band3 $f_{C R E}$ vs. estimated counterparts. Black dash line denotes the 1:1 slope. 

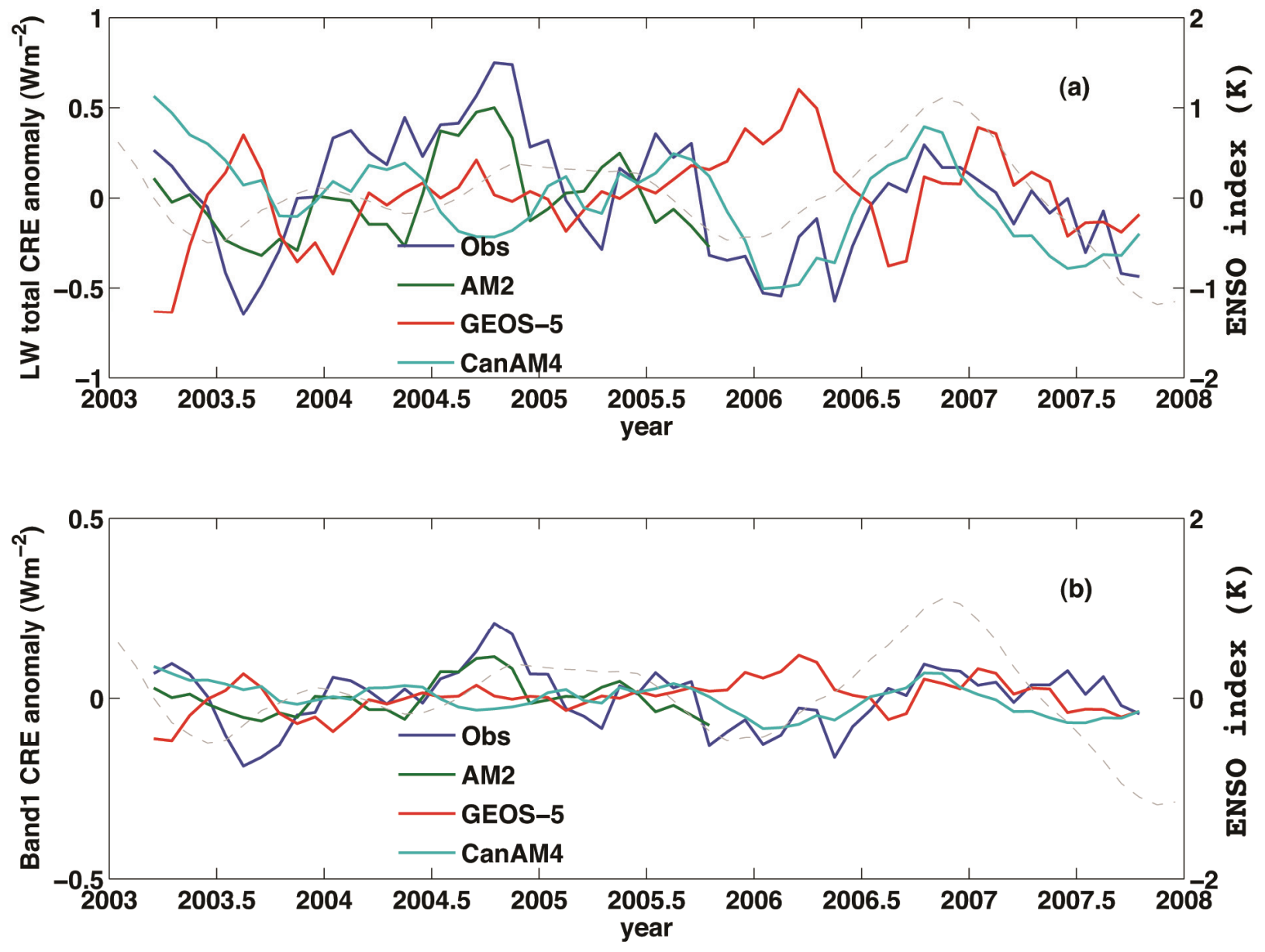

Figure 8. (a) 5-month running means of deseasonalized anomalies of total LW CRE from the observation (blue line), the GFDL AM2 (green line), NASA GEOS-5 output (red line), and CCCma CanAM4 (cyan line) simulations. The ENSO index is plotted in gray dash line. (b) Same as (a) except for the Band1 CRE instead of total LW CRE. Following previous studies, the ENSO index is defined as 5-month running mean of the SST anomalies averaged over the region of $5^{\circ} \mathrm{S}-5^{\circ} \mathrm{N}$, the South American coast and $180^{\circ} \mathrm{W}$. 


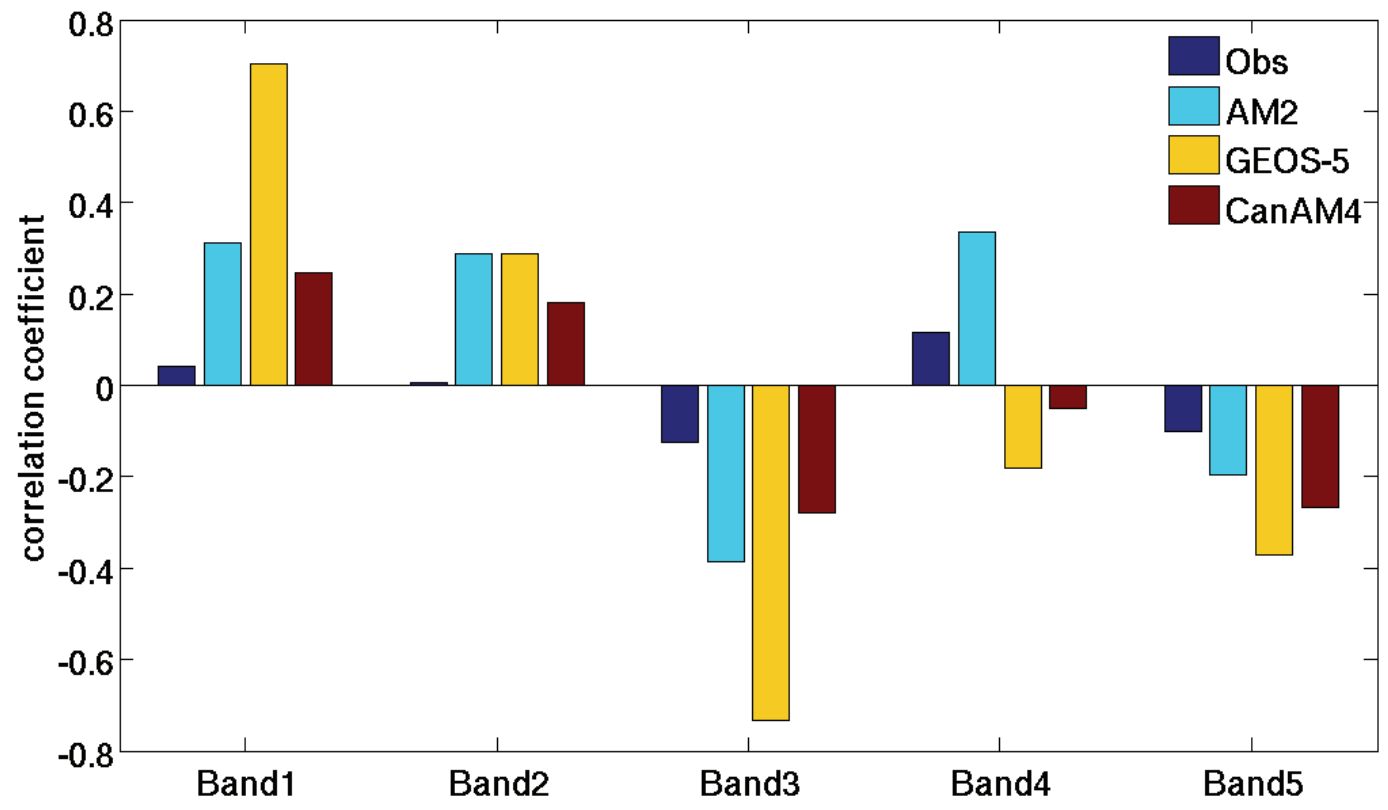

Figure 9. Correlation coefficients between the ENSO index and the 5-month running means of deseasonalized $f_{C R E}$ of each band. Results from observation and three models are shown in different colors. 


\section{Observation (Band 1)}
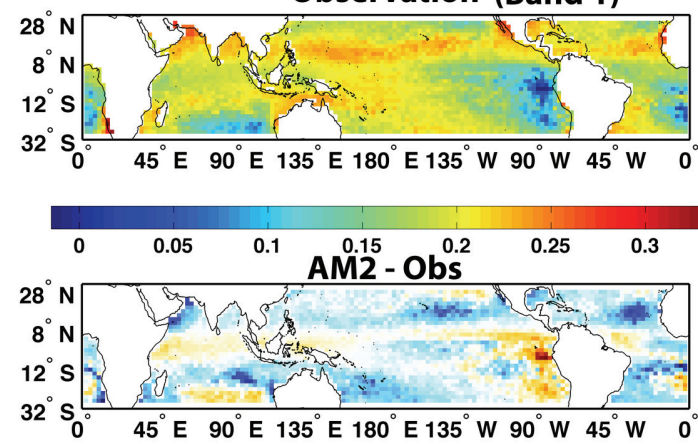

GEOS5 - Obs

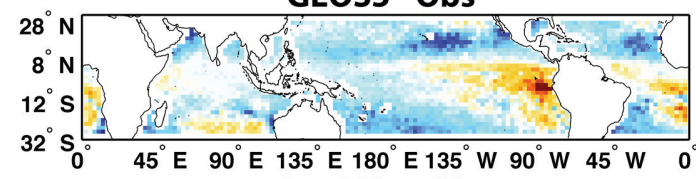
CanAM4 - Obs

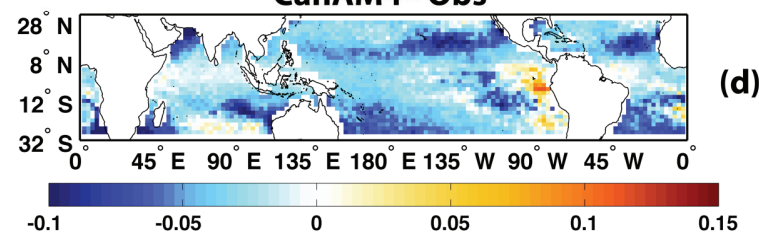

(a)

(c)

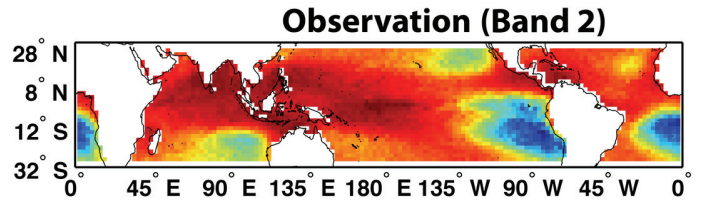

(e)

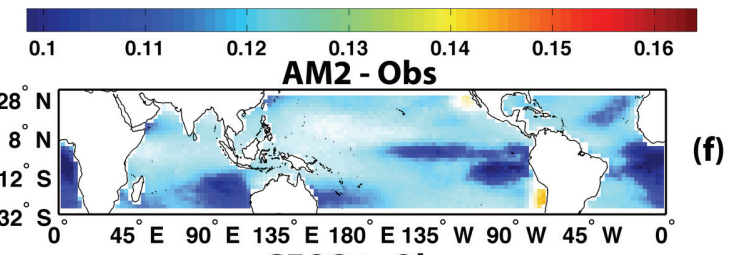

GEOS5 - Obs

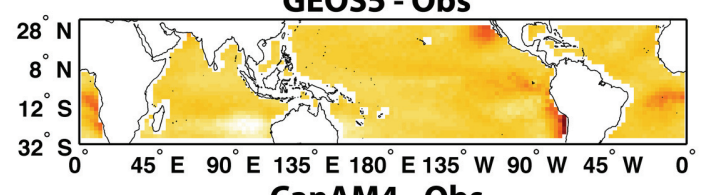

(g)

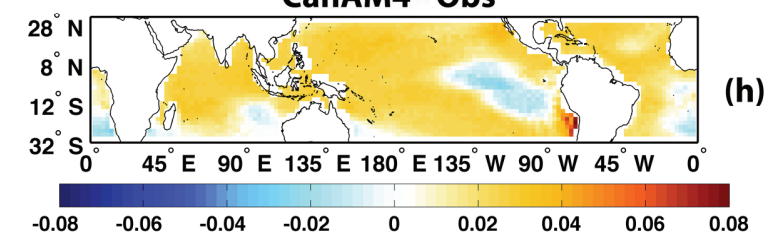

Figure 10 (a) Map of observed long-term mean of Band1 $f_{\text {CRE. }}(b)$-(d) Maps of model-observation difference in the long-term mean of Band1 $f_{C R E}$ for the GFDL AM2, NASA GEOS-5, and CCCma CanAM4 simulations, respectively. Note the same color scale is used for (b)-(d). (e) same as (a) except for Band $2 f_{\text {CRE. }}(f)-(h)$ same as $(b)-(d)$ except for Band $2 f_{C R E}$. 


\section{Observation (Band 3)}
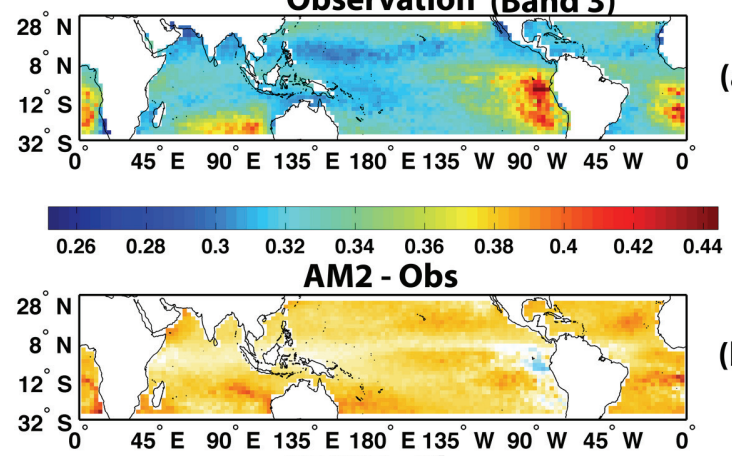

GEOS5 - Obs

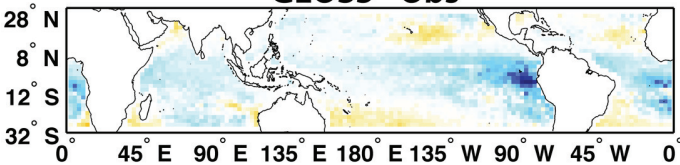
CanAM4 - Obs

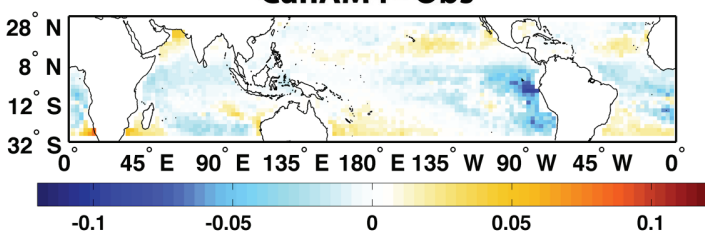

(a)

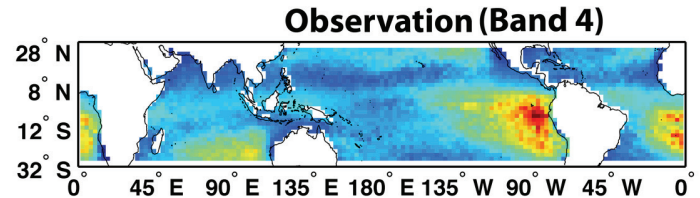

(e)

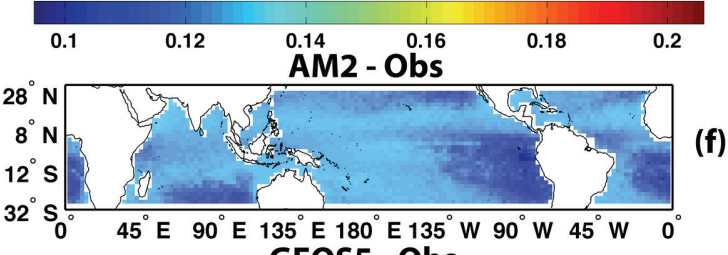

GEOS5 - Obs

(c)

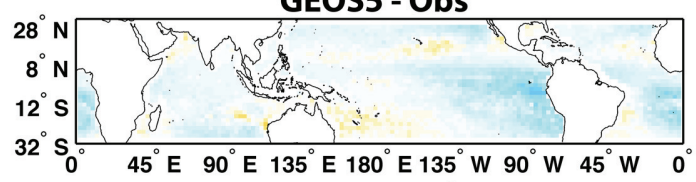

(g)

(d)

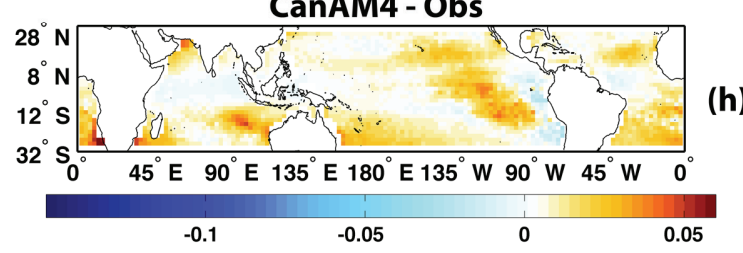

Figure 11. Same as Figure 10 except for Band3 $f_{C R E}$ and Band4 $f_{C R E}$. 

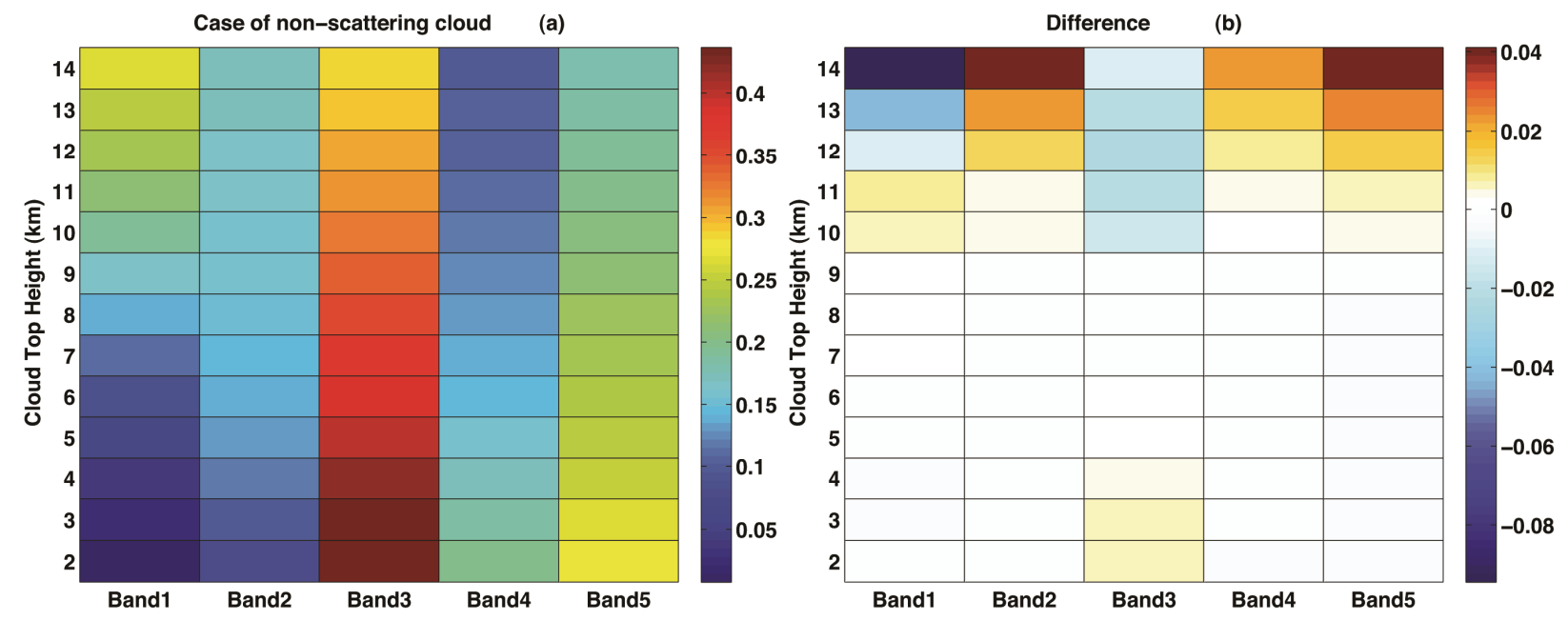

Figure 12. (a) Band-by-band $\mathrm{f}_{\mathrm{CRE}}$ for different cloud top height $(2 \mathrm{~km}-14 \mathrm{~km})$. Clouds are assumed to be opaque and non-scattering. (b) The difference in $f_{C R E}$ between the case of scattering cloud and the case of non-scattering cloud when everything else remains the same. 